\title{
Recent Trends in the Use of Statistical Tests for Comparing Swarm and Evolutionary Computing Algorithms: Practical Guidelines and a Critical Review
}

\author{
J. Carrasco ${ }^{1}$, S. García ${ }^{1}$, M.M. Rueda ${ }^{2}$, S. Das ${ }^{3}$, and F. Herrera ${ }^{1}$ \\ ${ }^{1}$ Department of Computer Science and AI, Andalusian Research Institute in Data \\ Science and Computational Intelligence, University of Granada, Granada, Spain \\ ${ }^{2}$ Department of Statistic and Operational Research, University of Granada, \\ Andalusian Research Institute of Mathematics, Granada, Spain \\ ${ }^{3}$ Electronics and Communication Sciences Unit, Indian Statistical Institute, 203 \\ B.T.Road, Kolkata 700108, West Bengal, India
}

February 24, 2020

\begin{abstract}
A key aspect of the design of evolutionary and swarm intelligence algorithms is studying their performance. Statistical comparisons are also a crucial part which allows for reliable conclusions to be drawn. In the present paper we gather and examine the approaches taken from different perspectives to summarise the assumptions made by these statistical tests, the conclusions reached and the steps followed to perform them correctly. In this paper, we conduct a survey on the current trends of the proposals of statistical analyses for the comparison of algorithms of computational intelligence and include a description of the statistical background of these tests. We illustrate the use of the most common tests in the context of the Competition on single-objective real parameter optimisation of the IEEE Congress on Evolutionary Computation (CEC) 2017 and describe the main advantages and drawbacks of the use of each kind of test and put forward some recommendations concerning their use.
\end{abstract}

Keywords statistical tests, optimisation, parametric, non-parametric, Bayesian

\section{Introduction}

Over the few last years the comparison of evolutionary optimisation algorithms and statistical analysis have undergone some changes. The classic paradigm consisted of the application of classic frequentist tests on the final results over a 
set of benchmark functions, although different trends have been proposed since then [1], such as:

- The first amendment after the popularisation of the use of statistical tests was the proposal of non-parametric tests that consider the underlying distribution of the analysed results, improving the robustness of the drawn conclusions [2].

- With this new perspective, some non-parametric tests [3, whose assumptions were less restrictive than the previous ones, were suggested for the comparison of computational intelligence algorithms [4].

- However, there are other approaches and considerations, like the convergence of the solution [5], robustness with respect to the seed and results over different runs and the computation of the confidence interval and confidence curves [6].

- As has already occurred in several research fields, a Bayesian trend [7] has emerged with some criticisms to the well known Null Hypothesis Statistical Tests (NHST) and there are some interesting proposals of Bayesian tests analogous to the classic frequentist tests [8].

Inferential statistics make predictions and obtain conclusions from data, and these predictions are the basis for the performance comparisons made between algorithms [9]. The procedure followed to reach relevant information is detailed below:

1. The process begins with the results of the runs from an algorithm in a single benchmark function. These results assume the role of a sample from an unknown distribution whose parameters we can just estimate to compare it with another algorithm's distribution.

2. Depending on the nature and purpose of the test, the results of the algorithms involved in the comparison will be aggregated in order to compute a statistic.

3. The statistic is used as an estimator of a characteristic, called parameter, of the distribution of interest, either the distribution of the results of our algorithm or the distribution of the algorithms' performance difference when we are comparing the results from a set of algorithms.

This procedure allows us to get information from experimental results, although it must be followed in order to obtain impartial conclusions which can also be reached by other researchers. Statistical tests should be considered as a toolbox to collect relevant information, not as a set of methods to confirm the previously stated conclusion. There are two main approaches: 
Frequentist These are the most common tests. Both Parametric and NonParametric tests follow this approach. Here, a non-effect hypothesis (in the sense of non-practical differences between algorithms) $\mathcal{H}_{0}$ and an alternative hypothesis $\mathcal{H}_{1}$ are set up and a test, known as Null Hypothesis Statistical Test (NHST) is performed. With the results of this test, we determine if we should reject the null hypothesis in favour of the alternative one or if we do not have enough evidence to reject the null hypothesis. This decision is made according to two relevant concepts in the Frequentist paradigm [10, 3, 11]:

- $\alpha$ or confidence coefficient: While estimating the difference between the populations, there is a certain confidence level about how likely the true statistic is to lie in the range of estimated differences between the samples. This confidence level is denoted as $(1-\alpha)$, where $\alpha \in$ $[0,1]$, and usually $\alpha=0.05$. This is not the same situation as if the probability of the parameter of interest lay in the range of estimated differences, but the percentage of times that the true parameter would lie in this interval if repeated samples from the population had been extracted.

- $p$-value: Given a sample $D$ and considering a null hypothesis $\mathcal{H}_{1}$, the associated $p$-value is the probability of obtaining a new sample as far from the null hypothesis as the collected data. This means that if the gathered data is not consistent with the assumed hypothesis, the obtained probability will be lower. Then, in the context of hypothesis testing, $\alpha$ represents the established threshold which the $p$-value is compared with. If the $p$-value is lower than this value, the null hypothesis is rejected.

- The confidence interval, the counterpart of the $p$-values, represents the certainty about the difference between the samples at a fixed confidence level. Berrar proposes the confidence curves, as a graphic representation that generalise the confidence intervals for every confidence level [6].

Bayesian Here, we do not compute a single probability but a distribution of the parameter of interest itself. With this approach, we avoid some main drawbacks of NHST, although they require a deeper understanding of the underlying statistics and conclusions are not as direct as in frequentist tests.

The main concepts of these families of statistical tests can be explained using Figure 1. In this figure we have plotted the density of distribution of the results of the different runs of two algorithms (DYYPO and TLBO-FL, which are presented in subsection 7.2 for a single benchmark. The density represents the relative probability of the random variable of the results of these algorithms for each value in the x-axis. In this scenario, a Parametric NHST would set up a null hypothesis about the means of both populations (plotted with a dotted 


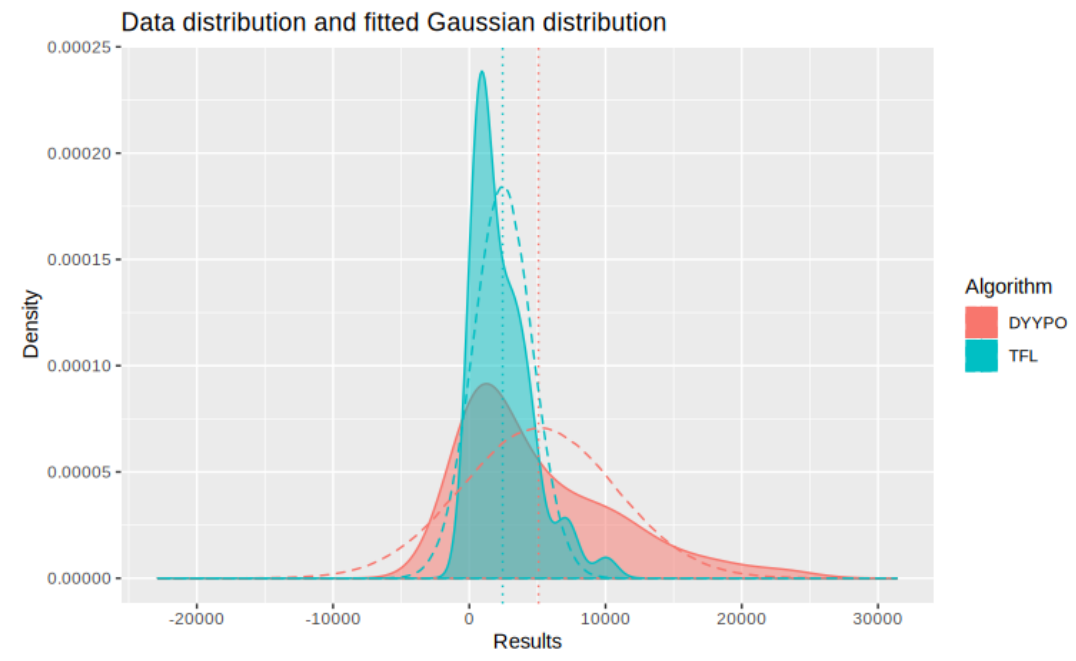

Figure 1: Comparison between real and fitted Gaussian distribution of results.

line), assuming that their distribution follows a Gaussian distribution. We have also plotted the Gaussian distributions with the mean and standard deviation of each population. In this context, there is a difference between the two means and the test could reject the null hypothesis. However, if we consider the estimated distributions, they are overlapped. This overlapping lead to the concept of effect size, which indicates not only if the population means can be considered as different, but quantifies this difference. In subsubsection 3.4.1 and Section 4 we will go in-depth in the study of this issue.

Nonetheless, there is a difference between the estimated Gaussian distributions and the real densities. This is the reason why Non-Parametric Tests arose as an effective alternative to their parametric counterparts, as they do not suppose the normality of the input data. In the Bayesian procedure, we would make an estimation of the distribution of the differences between the algorithms' results as we have made with the results themselves in Figure 1. Then, with the estimated distribution of the parameter of interest, i. e. the difference between the performances of the algorithms, we could extract the desired information.

Bayesian paradigm makes statements about the distribution of the difference between the two algorithms, which can reduce the burden of the researchers the NHST do not find significant differences between the algorithms.

Other significant concepts in the description of statistical tests come with the comparison of the tests themselves. Type I error occurs when $\mathcal{H}_{0}$ is rejected but it should not be. In our scenario, this means that the equivalence of the algorithms has been discarded although there is not a significant difference between them. Otherwise, type II error arises when there is a significant difference but this has not been detected. We denote alpha as the probability of making a type I error (often called significance level or size of test) and beta as the prob- 
ability of make a type II error. The power of a test is $1-\beta$, i. e. the probability of rejecting $\mathcal{H}_{0}$ when it is false, so we are interested in comparing the power of the tests, because with the same probability of making a type I error, the more powerful a test is, the more differences that will be regarded as significant.

There is some notation that is used along this work and it is summarised in Table 1

\begin{tabular}{|c|c|c|}
\hline Notation & Description & Location \\
\hline $\mathcal{H}_{0}$ & Null hypothesis & Section 3 \\
\hline $\mathcal{H}_{1}$ & Alternative hypothesis & Section 3 \\
\hline $\mathcal{H}_{j}$ & $\begin{array}{l}\text { When comparing multiple algorithms, each } \\
\text { one of the multiple comparisons. }\end{array}$ & Subsection 3.3 \\
\hline $\mathcal{H}_{i, j}$ & $\begin{array}{l}\text { When comparing multiple algorithms, the } \\
\text { comparison between the algorithm } i \text { and } j \text {. }\end{array}$ & Subsection 3.3 \\
\hline$\alpha$ & Significance level & Section 3 \\
\hline$\mu$ & Mean of the performance of an algorithm & Section 3.1 \\
\hline$k$ & Number of compared algorithms & Sections 3,5 \\
\hline$N_{m}(\mu, \Sigma)$ & $\begin{array}{l}\text { Multivariate Gaussian distribution, with pa- } \\
\text { rameters } \mu \text { and } \Sigma\end{array}$ & Section 3 \\
\hline$n$ & Number of benchmark functions & Sections 35 \\
\hline$\theta$ & Probability of stated hypothesis & Section 5 \\
\hline Dir & Dirichlet distribution & Section 5 \\
\hline DP & Dirichlet Process & Section 5 \\
\hline$\alpha$ & Prior distribution of DP & Section 5 \\
\hline $\mathcal{E}$ & $\begin{array}{l}\text { The expectation with respect to the Dirichlet } \\
\text { Process. }\end{array}$ & Subsection 5.3 \\
\hline $\begin{array}{r}\delta_{z} \\
I_{[\text {precondition }]}\end{array}$ & $\begin{array}{l}\text { Dirac's delta centered in } z \\
\text { Indicator function. } \quad \text { Takes } 1 \text { when } \\
\text { precondition is fulfilled, } 0 \text { otherwise. }\end{array}$ & \begin{tabular}{|l|} 
Section 5 \\
Section 5
\end{tabular} \\
\hline
\end{tabular}

Table 1: General notations used in this paper

In this paper, we gather the different points of view of the statistical analysis of results in the computational intelligence research field with the associated theoretical background and the properties of the tests. This allows comparisons to be made regarding the appropriateness of the use of each testing paradigm, depending on the specific situation. Moreover, we also adapt the calculation of these confidence curves with a non-parametrical procedure [12], as it is more convenient in the context of the comparison of evolutionary optimisation algorithms.

We have included in this paper the performance and the analyses of the tests in the context of the 2017 IEEE Congress on Evolutionary Computation (CEC) Special Session and Competition on Single Objective Real Parameter Numerical Optimisation [13]. This case study allows for a clear illustration of the use of these tests, their behaviour with different distributions of data and the conclusions that can be made with their outputs. The test-suite proposed for this 
competition can be considered to be a relevant benchmark for the comparison of any newly proposed algorithms. Moreover, the results achieved and final rankings of this competition are supposed to indicate what is the state-of-the-art in evolutionary computation and against which algorithms our proposals should be compared.

The code for the tests and the analysis, tables and plots are included as a vignette in the developed $\mathrm{R}$ package rNPBST 1 14]. We have also developed a shiny application to facilitate the use of the aforementioned tests. This application processes the results of two or more algorithms and performs the selected test. The results are exported in TEXformat and an HTML table. Cases in which there is a plot associated with the test are highlighted.

This paper is organised as follows. In Section 2 we include a survey on the main statistical analyses proposed in the literature for evaluating the classification and optimisation algorithms. Section 3 contains a depiction of the well-known parametric tests and non-parametric tests respectively. In Section 4 we describe the main criticisms and proposals concerning the traditional tests. Section 5 introduces Bayesian test concepts and notations. Due to the relevance of the Multi-Objective problem in the optimisation scientific community, we have gathered the tests that address this issue in Section 6 regardless of their statistical nature. In Section 7, we describe the setting of the CEC'2017 Special Session, which is performed in Section 8 and summarised in Section 9. The lessons learnt and test considerations are presented in Section 10. Section 11 summarises the conclusions obtained in the analyses.

\section{Survey on Statistical Analyses Proposed}

In this section, we provide a chart with an extensive survey on the different statistical proposals made for the comparison of machine learning and optimisation algorithms. The survey is included in Table 2. This table includes a categorisation of the methodologies according to their statistic nature, a brief description of the underlying idea and the considered scenario of the comparison of the data.

The proposals are sorted by the year of publication. This order highlights past and present trends in the statistical comparison. The first proposals were made from a frequentist and fundamentally parametric point of view. Later, with the work of Demšar [15] non-parametric tests arose as the alternative to parametric ones in certain circumstances. In recent years, some Bayesian proposals were made, although they still depend on the results' distributions and are focused on the comparison of classifiers.

Moreover, most of the proposals are oriented to compare classifiers. The tests and guidelines made for the comparison of optimisation algorithms are consistent and are constantly used in the literature. However, there is not a specific test that takes account of the multiple runs in the same benchmark

${ }^{1}$ https://github.com/JacintoCC/rNPBST 
function and estimates the correlation between these runs, as the tests suggested for cross-validation setups do. 


\begin{tabular}{|c|c|c|}
\hline Citation & Kind of test & Description \\
\hline 16 & Non-parametric - Classification & Cochran Test for the distribution of mistakes on the classification of $n$ samples. \\
\hline 17 & Parametric - Classification & $\begin{array}{l}\text { McNemar Test and } t \text {-test variants for different scenarios of the comparison of } \\
\text { two classifiers accuracy. }\end{array}$ \\
\hline 18 & Parametric - Classification & Proposal of $5 \times 2 \mathrm{cv} \mathrm{F}$ test. \\
\hline 19] & Parametric - Optimisation & ANOVA test for parameter analysis in genetic algorithms. \\
\hline 20 & Frequentist - Classification & Introduces corrections for multiple testing (Bonferroni, Tukey, Dunnet, Hsu). \\
\hline 21 & Parametric - Classification & Proposal using variance estimators of cross-validation results. \\
\hline 22 & Non-parametric - Classification & Proposal of multiple comparisons using Cochran test. \\
\hline 23] & Parametric - Optimisation & Guide on the use of ANOVA test in exploratory analyses of genetics algorithms. \\
\hline 24 & Parametric - Classification & $\begin{array}{l}\text { Computation of the sample size for a desired confidence interval width of the } \\
\text { True Positive Rate and True Negative Rate. }\end{array}$ \\
\hline 25 & Parametric - Classification & Proposal of MultiTest algorithm that order the competitors using post-hoc test. \\
\hline 15 & Frequentist - Classification & $\begin{array}{l}\text { Review of the use of previous parametric tests, Wilcoxon Signed-Rank and } \\
\text { Friedman test. }\end{array}$ \\
\hline 26$]$ & Frequentist - Information Retrieval & Comparison of pairwise non-parametric tests with Student's paired t-test. \\
\hline 2 & Frequentist - Classification & Criticisms and tips on the use of statistical tests. \\
\hline 27 & Non-parametric - Classification & Extensive proposal of non-parametric tests and associated post-hoc methods. \\
\hline [28] & Parametric - Classification & $k$-fold cross validated paired $t$-test for AUC values. \\
\hline 4 & Non-parametric - Optimisation & $\begin{array}{l}\text { Study of the preconditions for a safe use of the parametric tests and proposal } \\
\text { of non-parametric methods for an optimisation scenario. }\end{array}$ \\
\hline 29] & Non-parametric - Classification & $\begin{array}{l}\text { Study of test application prerequisite in a classification context and with dif- } \\
\text { ferent measures. }\end{array}$ \\
\hline 30 & Non-parametric - Classification & Study on the use of statistical tests in neural networks' results. \\
\hline 31 & Non-parametric - Classification & $\begin{array}{l}\text { Newly proposed post-hoc methods and non-parametric comparison (Li, Holm, } \\
\text { Holland, Finner, Hochberg, Hommel and Rom post-hoc procedures). }\end{array}$ \\
\hline 32 & Parametric - Classification & Repeated McNemar's test. \\
\hline 33 & Frequentist - Classification & Decomposition of the variance of the $k$-fold CV for prediction error estimation. \\
\hline 34 & Permutational - Classification & $\begin{array}{l}\text { Presentation of two permutational tests that study if the algorithm has learnt } \\
\text { the data structure and if it uses the attributes distribution and dependencies. }\end{array}$ \\
\hline 35 & Parametric - Optimisation & $\begin{array}{l}\text { A multicriteria comparison algorithm (MCStatComp) using the aggregation of } \\
\text { the criteria through a non-dominance analysis. }\end{array}$ \\
\hline 36 & Non-parametric - Optimisation & Tutorial on the use of non-parametric tests and post-hoc procedures. \\
\hline 37] & Non-parametric & $\begin{array}{l}\text { Review on the use of non-parametric tests and post-hoc tests, and the impact } \\
\text { of normality. }\end{array}$ \\
\hline 38 & Non-parametric - Classification & $\begin{array}{l}\text { Multi }{ }^{2} \text { Test algorithm, which orders the algorithms using non-parametric pair- } \\
\text { wise tests. }\end{array}$ \\
\hline 39 & Non-parametric - Classification & Survey on the use of statistical tests in Bioinformatics field. \\
\hline 40 & Bayesian - Classification & Bayesian Poisson binomial test for pairwise comparison of classifiers. \\
\hline 41$]$ & Bayesian - Classification & Hierarchical study through Bayesian inference. \\
\hline 42 & Parametric - Classification & Inclusion of a certain level of allowed error in paired $t$-test. \\
\hline [4] & Parametric - Classification & Comparison using McNemar test with different measures. \\
\hline 44] & Permutation - Classification & Permutation (bootstrap) tests for a cross-validation setup. \\
\hline 45] & Parametric - Classification & Blocked $3 \times 2$ cross validation estimator of variance. \\
\hline [5] & Non-parametric - Optimisation & Analysis of convergence using Page test. \\
\hline 46] & Non-parametric - Classification & Proposal of Page test for parameter trend study. \\
\hline 47 & Bayesian & Bayesian version of Wilcoxon Signed Rank test. \\
\hline 48 & Bayesian & Bayesian test without prior information using Imprecise Dirichlet Process. \\
\hline 49 & Bayesian - Classification & $\begin{array}{l}\text { Bayesian test for two classifiers on multiple data sets accounting the correlation } \\
\text { of cross-validation. }\end{array}$ \\
\hline [50] & Bayesian & Proposal of Multiple Measures tests. \\
\hline 51 & Bayesian & Presentation of the Bayesian Friedman test. \\
\hline 52 & Parametric - Information retrieval & Confidence interval for $F_{1}$ measure using blocked $3 \times 2$ cross validation. \\
\hline 53 & Non-parametric - Classification & Generalisation of Wilcoxon rank-sum test for interval data. \\
\hline 54 & Non-parametric - Classification & $\begin{array}{l}\text { Proposal off Mann-Whitney U test for two classifiers and the Kruskal-Wallis } \mathrm{H} \\
\text { test for multiple classifiers with the associated post-hoc corrections. }\end{array}$ \\
\hline 55 & Frequentist - Classification & Proposal of Wald and Score tests for precision comparison. \\
\hline 56 & Bayesian - Classification & $\begin{array}{l}\text { Bayesian hierarchical model for the joint comparison of multiple classifiers on } \\
\text { multiple data sets with the cross-validation results. }\end{array}$ \\
\hline 6] & Parametric - Classification & Presentation of the confidence curves as the confidence interval generalisation. \\
\hline 57 & Non-parametric - Classification & Proposal of exact computation of Friedman test. \\
\hline 8 & Bayesian & Extensive tutorial on the use of Bayesian tests. \\
\hline 58 & Non-parametric - Classification & New proposals of non-parametric tests that introduce weights. \\
\hline 59 & Frequentist - Optimisation & $\begin{array}{l}\text { Application of Deep Statistical Comparison of Multi-Objective Optimisation } \\
\text { algorithms for an ensemble of quality indicators. }\end{array}$ \\
\hline 60 & Bayesian & Bayesian analysis based on a model over the algorithms' rankings. \\
\hline 61 & Parametric - Classification & Methodology for the definition of the sample sizes. \\
\hline 62 & Frequentist - Optimisation & $\begin{array}{l}\text { Extension of Deep Statistical Comparison, a two-step comparison that select } \\
\text { the appropiate parametric or non-parametric test according to the normality } \\
\text { of the data. }\end{array}$ \\
\hline
\end{tabular}

Table 2: Survey on different statistical proposals for results analysis 


\section{$3 \quad$ Frequentist tests}

In this section, we describe the classic frequentist tests: the properties of the parametric tests and their assumptions, and the different non-parametric tests and their application in the context of the comparison of single-objective and bound-constrained evolutionary optimisation algorithms. Although there are other tests, algorithms and proposals, as reflected in Table 2 , the tests presented in this section represent the core of the statistical comparison methodology.

\subsection{Parametric Statistical Tests}

Parametric tests make the assumption that our sample comes from a distribution that belongs to a known family, usually the Gaussian family, and it is described with a little number of parameters [3].

t-test This classic test is used to compare two samples. Null hypothesis consists in the equivalence of the means of both populations. The main assumptions made by t-test is that the samples have been extracted randomly and the distribution of the populations of the samples are normal.

The required input of this test is the group of observations of the different runs of the pair algorithms that will be compared for a single problem.

Analysis of Variance When we are interested in the comparison of $k$ distinct algorithms, we need another test, because repeating the t-test for every pair of algorithms would increment the type I error. The Analysis of Variance (ANOVA) test of the null hypothesis consists in the equivalence of all the means:

$$
\mathcal{H}_{0}: \mu_{1}=\cdots=\mu_{k}
$$

against $\mathcal{H}_{1}: \exists i \neq j, \mu_{i} \neq \mu_{j}$. ANOVA test deals with the variance within a group, between groups or a combination of the two types.

Here the input consists of a matrix where each column represents an algorithm and each row is a single benchmark function, while the cells contain the mean of the performance for all runs of each algorithm in each benchmark [19].

These tests are very relevant in the statistical comparison, although they have troublesome prerequisites in the field of comparison of optimisation algorithms. Then, non-parametric statistical tests were proposed to address this issue with a known methodology.

\subsection{Non-Parametrical Statistical Tests}

According to Pesarin [63], $\mathcal{P}$ is a non-parametric family of distributions if it is not possible to find a finite-dimensional space $\Theta$ in which there is a one-to-one relationship between $\Theta$ and $\mathcal{P}$. This means that we do not have to assume that the underlying distribution belongs to a known family of distributions. Consequently, the prerequisites for non-parametric tests such as symmetry or 
continuity, are less restrictive than parametric ones and non-parametric tests are more robust and less sensitive to dirty data [15, 27].

\subsubsection{Check of the preconditions}

The goodness of fit tests, for example, Kolmogorov-Smirnov, Shapiro-Wilk or D'Agostino-Pearson, are used to determine whether the normality of the distribution can be rejected [3, 4]. This can be used to assure a safer use of parametric tests like ANOVA or t-test. Another usual prerequisite of parametric tests is the homoscedasticity, that is, the equivalence of the variances of the populations. Levene's test is used to this purpose, so that we can reject the hypothesis of the equivalence of the variances at a level of significance, and then one of the alternatives would be to use a non-parametric test [64, 29]. Although it is correct to use non-parametric tests to achieve our main goal before even checking these preconditions, it is worth doing it because parametric tests make more assumptions about the population and when they are satisfied they have stronger information and consequently in these circumstances they are more powerful.

However, non-parametric tests also make some assumptions that are easier to fulfil than parametric tests, like symmetry in the Wilcoxon Signed-Ranks test. This means that a safe use of this test is depending on the symmetry of the population, i. e. the probability density function is reflected around a vertical line at some value 65.

\subsubsection{Pairwise comparisons}

The first kind of experimental study would consist in the comparison of a pair of algorithms' performance in several benchmarks. A pair of non-parametric tests are presented in this section.

Sign-test This simple test developed by Dixon and Mood [66] gives an idea of the way that non-parametric tests are developed. The assumption made here is that if the two algorithms involved in the comparison had an equivalent performance, the number of cases where each algorithm outperforms the other would be similar.

Wilcoxon signed-rank test The underlying idea of this test [67] is the next step in non-parametric tests, not just making a count of the wins of each algorithm but ranking the differences between the performance and developing the statistic over them [68]. This is already a widespread test used to compare two algorithms when t-test prerequisites are not fulfilled.

These tests receive the means of the runs for each benchmark function of two algorithms. Unlike the parametric tests, in which null hypothesis consists in the equivalence of the means, in these tests $\mathcal{H}_{0}$ is the equivalence of the medians. 


\subsubsection{Multiple comparisons}

Sometimes we are interested in the joint analysis of multiple algorithms. We have the result of every algorithm for every problem, but we are not allowed to make a pairwise comparison for each pair of algorithms without losing the control on the Family-Wise Error Rate (FWER), i. e. augmenting the probability of making at least one type I error. This effect is easily computed. If we allow an error of $\alpha$ in each test (the significance) and conduct $k$ tests, the probability of making at least one error would be $1-(1-\alpha)^{k}$. Some methods are described below so that they can be used instead of repeated pairwise comparisons:

Multiple Sign test This test is an extension of Sign test when we compare $k$ algorithms with a control algorithm. Differences for every pair (algorithm, problem) with the control algorithm are computed and the number of each sign is counted. Then, the median of the performance of every algorithm is compared with the median of the control algorithm. A directional null hypothesis is rejected if the number of minus (or plus) signs is less than or equal to the corresponding critical value available in the table for a treatment versus control multiple comparisons sign test [69] for specific values of $n$ (the number of benchmarks), $m=k-1$ and level of significance $\alpha$.

Friedman test Friedman test is the non-parametric test that accomplishes the ANOVA test purpose. The null hypothesis in this test is the equivalence of the medians of the different algorithms and the alternative hypothesis consists in the difference of two or more algorithms medians. To calcu-

late the statistic, we rank the algorithm performance for each problem and compute the average of each algorithm between problems. Iman and Davenport proposed a more powerful version of this test using a modified statistic [70].

Friedman Aligned-Ranks test The main inconvenient of the Friedman test is that ranks are just considered in each problem, which leads to a conservative test when comparing few algorithms. However, Friedman AlignedRanks test [71] considers every algorithm-problem pair to produce a rank from 1 to $k \cdot n$.

Quade test The Quade test [72] takes into account the different difficulties of the problems. This is made calculating the range of the problems as the maximum differences between the samples and ranking the $n$ ranges. Then, these ranks are assigned to the problems and represent a weight to the ranks obtained by the Friedman method. With these weighted ranks, the procedure follows as usual.

\subsubsection{Comparison of Convergence Performance}

A fundamental aspect that should be considered in the comparison of optimisation algorithms is the convergence of their results, as it is a desirable property. 
Derrac et al. suggest the use of the Page trend test for this purpose [5]. In the original definition of the Page test, this test is designed to detect increasing trends in the rankings of the original data. The idea used for the comparison of the convergence is to apply the test in the differences of the results between two algorithms $\mathbf{A}$ and $\mathbf{B}$ at $c$ equidistant cut points of the search on the corresponding $n$ benchmark functions. The null hypothesis is the equivalence of the means for each cut point and this hypothesis is rejected in favor of an ordered alternative, which states that there is an increasing trend in the ranks of the differences between $\mathbf{A}$ and $\mathbf{B}(\mathbf{A}-\mathbf{B})$. Under these preconditions and assuming that the objective is the minimisation of the functions, the interpretation to be made is as follows:

1. If $\mathcal{H}_{0}$ is rejected, then an increasing trend is detected. This could mean that the optimum of $\mathbf{A}$ is growing faster than the result of the $\mathbf{B}$ algorithm (which lacks of logic because when an algorithm finds a minimum, it does not grow with the iterations) or the fitness of $\mathbf{B}$ is decreasing faster than the fitness of $\mathbf{A}$, that is, the algorithm $\mathbf{B}$ converges faster than the algorithm A.

2. If the test is made over $\mathbf{B}-\mathbf{A}$ and the null hypothesis is rejected, the reasoning is analogous to the previous one and the algorithm $\mathbf{A}$ converges faster.

3. If $\mathcal{H}_{0}$ is not rejected, we cannot say anything about the relative convergence of the two compared algorithms.

As we are analyzing the differences in the trend between the algorithms $\mathbf{A}$ and $\mathbf{B}$, a modification in the rankings of the difference is needed when an algorithm reaches the optimum before the end depending on the circumstance:

- If no algorithm reaches the optimum, there is not any further change.

- If algorithm A reaches the optimum before the end, ranks should increase from the cut point where algorithm $\mathbf{A}$ reaches the optimum to the last cut point in a way that the highest ranks are in the last positions.

- If algorithm $\mathbf{B}$ reaches the optimum before the end, ranks should decrease from the cut point where algorithm $\mathbf{B}$ reaches the optimum and include the lowest values.

- If both algorithms reach the optimum at the same point, the ranks are computed as normal in the original version of the test.

It is important to note that this test focuses on the trends of the differences, so the conclusions are submitted to the sign of the difference between the firsts observations. This means that if the algorithm $\mathbf{A}$ starts at a point near the objective and then decrease its score function slowly and algorithm $\mathbf{B}$ starts with a bad score, the test could reject the null hypothesis because the algorithm $\mathbf{B}$ reduce its score at a greater pace. Then, the comparison between the 
convergences should be made in the context of algorithms with similar initial performance, where the convergence is the key aspect of the performance.

Another relevant issue of the Page test is that we can only compare two algorithms at a time. Then, if we are interested in the comparison of the convergence (or final results) of multiple algorithms or the fine-tuning of the parameters, we should incorporate the post-hoc procedures to the statistical background described in this paper.

\subsection{Post-hoc Procedures}

We should, however, be conscious that these multiple-comparison tests, as in ANOVA, only can detect significant differences between the whole group of algorithms, but cannot tell where these differences are. Then, we declare a family of hypotheses of the comparison between a control algorithm and the $k-1$ remaining algorithms or among the $k$ algorithms, with $k-1$ or $k(k-1) / 2$ hypotheses, respectively. Post-hoc tests aim to obtain a set of $p$-values for this family of hypotheses.

We compute the $p$-value of every hypothesis using a normal approximation for a statistic that depends on the non-parametric test used. However, as we previously stated, singular $p$-values should not be used for multiple comparison due to the loss of the control over the FWER, so an adjustment is made to obtain the adjusted $p$-values (APVs). Several methods are available for the computation of the adjusted $p$-values are for example Bonferroni-Dunn, Li, Holm, Holland, Hochberg and Rom methods [31].

\subsubsection{One vs. $n$ algorithms}

In the context of a comparison of multiple algorithms versus one control algorithm, we can follow the Bergmann and Hommel procedure, where we say that an index set of hypotheses $I \subseteq\{1, \ldots, m\}$ is exhaustive if all associated hypothesis $H_{j}, j \in I$ could be true. It rejects every hypothesis $H_{j}$ as $j$ is not included in the acceptance set $A=\cup\left\{I: I\right.$ exhaustive, $\left.\min \left\{p_{i}: i \in I\right\}>\alpha /|I|\right\}$, where $|\cdot|$ represents the cardinality of a set. Due to the high cost of checking if each $I$ is exhaustive, a set $E$ with all the exhaustive sets of hypotheses is previously calculated and then the hypotheses that are not in $A$ are rejected. For the Bergmann-Hommel test, $A P V_{i}=\min \{v, 1\}$ where $v=\max \left\{|I| \cdot \min \left\{p_{j}, j \in\right.\right.$ $I\}: I$ exhaustive, $i \in I\}$.

\subsection{2 $n$ vs. $n$ algorithms}

Although post-hoc procedures provide an adjusted and reliable $p$-value for the comparison between algorithms even when we are making the comparison among all the algorithms, we must consider that not all combinations of true and false in the hypotheses are allowed. For example, if the hypothesis of the equivalence between algorithms $i$ and $j$, say $H_{i, j}$ is rejected, at least one of the hypotheses $H_{i, k}$ and $H_{j, k}$ must be also rejected. 
When we are interested in carrying out all $m=\frac{k(k-1)}{2}$ pairwise comparisons, we can simply use the Holm's test (with a little modification) or the Nemenyi procedure. These variants make an adjustment on the signification level through the computed $p$-values: Holm $p$-value is computed as $A P V_{i}=\min \{v, 1\}$, with $v=\max \left\{(m-j+1) p_{j}: 1 \leq j \leq i\right\}$; and Nemenyi $A P V_{i}=\min \{v, 1\}$, where $\left.v=m p_{i}\right)$. The latter procedure is equivalent to if $\alpha$ was divided by the number of comparisons performed $(m)$. There is an associated graphic with this test proposed by Demšar, known as Critical Difference plots [15], where the algorithms are ordered by their mean ranks and linked when the null hypothesis of their equivalence is not rejected by the test. Then, in a single plot, it can be seen what algorithms equivalence is discarded by the post-hoc procedure and which groups are formed with a not sufficiently different performance. This method is simple but is less powerful than the other ones [36]:

Shaffer's static procedure This method is based on the Holm's test although it takes into account the possible hypotheses that can be simultaneously true. Obtained $p$-values are ordered and hypotheses $H_{1}, \ldots, H_{i}$ are rejected if $p_{i}<\alpha / t_{i}$, with $t_{i}$ the maximum number of hypotheses that can be true when any of $H_{1}, \ldots, H_{i}$ are false. This method does not depend on the $p$-values but it is determined by the given hypotheses. $t_{i}$ values are the maximum of the set

$$
S(k)=\bigcup_{j=1}^{k}\left\{\left(\begin{array}{l}
j \\
2
\end{array}\right)+x: x \in S(k-j)\right\} \text { if } k>2, S(0)=S(1)=\{0\},
$$

with $S(k)$ the set of numbers of hypotheses which can be true when $k$ algorithms are compared.

Shaffer's dynamic procedure This is a modification to increase the power of the method. $\alpha=t_{i}$ is substituted by $\alpha=t_{i}^{*}$, the maximum number of hypothesis that can be true with the current assignment. That means that $t_{i}^{*}$ also depends on the already rejected hypothesis. Shaffer $A P V_{i}=$ $\min \{v, 1\}$ where $v=\max \left\{t_{j} p_{j}: 1 \leq j \leq i\right\}$.

\subsection{Confidence Intervals and Beyond}

Confidence Intervals (CIs) are usually used in the context of statistical analysis, presented as a counterpart for the $p$-value. As classic NHST, CIs are defined at an arbitrary although fixed level of confidence (usually $95 \%$ ), but they represent a suitable range for the location of the parameter of interest. The information given by the effect size concept is then included in the width of the CI: a narrower interval implies more certainty about the real difference. CI also includes the null-hypothesis test information. In general, if a 95\%-CI does not include the value that represents the equivalence of the performance, the NHST gives a $p$-value less than 0.05 and the hypothesis is rejected [73]. On the other hand, if the null value belongs to the $95 \%$-CI, the equivalence of the algorithms cannot be discarded. 
Although in the Berrar's work [73] the criticisms are applicable for NHST for both classification and optimisation problems, the proposal of the computation of the confidence intervals described in this paper is not valid for optimisation problems because it expects the data to come from a cross-validation experiment.

Next, we propose here a modification over the computation of the confidence intervals from a non-parametric and ranking perspective, adducing the reasons that justify the use of the Wilcoxon test but without the drawbacks of NHST [12.

If we have $l$ observations from two algorithms over one benchmark function, $\left\{x_{1}, \ldots, x_{l}\right\},\left\{y_{1}, \ldots, y_{l}\right\}$, then we estimate the approximate $100(1-\alpha) \%$ confidence interval of the difference of the two medians with the median of all the $l^{2}$ differences with the following process:

1. $K=W_{\alpha / 2}-\frac{l(l+1)}{2}$, where $W_{\alpha / 2}$ is the $100 \alpha / 2$ percentile of the Wilcoxon two-sample test statistic distribution (see pp 156-162 of Center [74]).

2. When sample size is greater than $20, K$ can be approximated by $K=$ $\frac{l^{2}}{2}-z_{1-\alpha / 2} \sqrt{\frac{l^{2}(2 l+1)}{12}}$, and then rounded to the next integer value, with $z_{1-\alpha / 2}$ is the $1-\alpha / 2$ percentile of the standard Gaussian distribution.

3. The confidence interval is defined from the $K$ th smallest difference to the $K$ th greatest of the $l^{2}$ differences of the sample.

\subsubsection{Confidence Curves}

Berrar undertook an in-depth study on the use of confidence intervals through the concept of the confidence curve [6], which is a plot of the confidence intervals for all levels for a point estimate, i. e. the observed difference of the samples. Thus, criticisms like the arbitrariness of the percentage of the level of significance are turned down.

In this confidence curve, the $x$-axis represents each null hypothesis while the $y$-axis shows the associated $p$-value. With this representation, for each level $\alpha$ in the $y$-axis, in the $x$-axis we obtain a $100(1-\alpha) \%$ confidence interval. Therefore, a wider curve means that there is less certainty about the estimated difference between the algorithms.

An interesting advantage of the use of confidence curves in the comparison of optimisation algorithms is the availability in a single comparison of the information given by an NHST (if the null hypothesis is rejected), the classic confidence interval (and the effect size), and the different confidence intervals at other significance levels. However, the information provided by this method requires more attention than a single $p$-value. 


\section{Known Criticisms to Null Hypothesis Statisti- cal Tests}

The need of the use of statistical tests in the analyses and experimentation is unquestioned. However, certain controversy exists regarding the types of these tests and the implications of their results, mainly from a Bayesian perspective [75]. The most repeated criticisms about the NHST are:

- They do not provide the answer that we expected [76, 8]. Commonly, when we are using statistical tests to compare optimisation algorithms, we want to prove that our algorithm outperforms existing algorithms. Then, once an NHST is carried out, a $p$-value is obtained and this is often misunderstood as the probability of the null hypothesis being true. As we previously mentioned, the $p$-value is the probability of getting a sample as extreme as the observed one, i. e. the probability of getting that data if the null hypothesis is true, $P\left(D \mid \mathcal{H}_{0}\right)$, instead of $P\left(\mathcal{H}_{0} \mid D\right)$.

- We can almost always reject $\mathcal{H}_{0}$ if we get enough data, making a little difference significant through a high number of experiments. This implies that a test could determine a statistically significant difference but without practical implications. On the other hand, a significant difference may not be detected when there is insufficient data. Usually when an NHST is made, all hypotheses with $p$-value $p \leq 0.05$ are rejected, and those with $p>$ 0.05 are considered not significant. This issue could lead to the inclusion in the experimentation of a new proposal of many benchmark functions to cause the rejection of the null hypothesis although the differences between the algorithms were random and very small.

- There is an important misconception related to the reproducibility of the experiments, as a lower $p$-value does not mean a higher probability of obtaining the same results if the experiments are replicated as is described by Berrar and Lozano [73]. This is because, if the null hypothesis is rejected, the probability of obtaining a significant $p$-value is determined by the power of the test, which depends on the $\alpha$ level, the true effect size in the target distribution and the size of the test set, but not on the $p$-value.

- A crucial issue is that we have no information when an NHST does not reject the null hypothesis and not finding a significant difference does not mean that the algorithms performance is equivalent.

\section{Bayesian Paradigm and Distribution Estima- tion}

In recent years, the Bayesian approach has been proposed as an alternative of frequentist statistics for comparing algorithms performance in optimisation and 
classification problems [8]. In the following we also describe some differences with respect to the NHST and their problems addressed in the previous section.

\subsection{Bayesian Parameter Estimation and Notation}

In this Bayesian approach, a set of candidate values of a parameter, which includes the possibility of no difference between samples, is set up [7]. Then we compute the relative credibility of the values in the candidate set according to the observations and using Bayesian inference. This approach is preferable in optimisation comparison to Bayesian model comparison approach, because this kind of comparison does not have a null value that brings the NHST objections back to Bayesian analysis.

Bayesian analysis is executed in three steps:

First step Establish a mathematical model of the data. In a parametric model it will be the likelihood function $P($ Data $\mid \theta)$.

Second step Establish the prior distribution $P(\theta)$. The common procedure is to select a prior distribution whose posterior distribution is known.

Third step Use Bayes' rule to obtain the posterior distribution $P(\theta \mid$ Data $)$ from the combination of likelihood and prior distribution. This means that we can see the distribution of the difference of performance between the algorithms, which may reveal that their results differ but that there is no algorithm that outperforms the other. For instance, if one algorithm gets better results in one problem, but worse in another one, using Frequentist statistics we would not reject the hypothesis of equivalence, but we could not know if their results are similar in all the observations.

The tests described in this section are oriented to obtain the posterior distribution of the difference of performance between two algorithms, noted as $z$. We set a prior Dirichlet Process (DP) with base measure $\alpha$. This process is a probability measure on the set of probability measures on a determined space (as we are interested in the distribution of $z$, this space will be $\mathbb{R}$ ). This means that if we make a sample from a DP, we do not obtain a number, like we would do if we make a sample from a uni-dimensional Gaussian distribution, but a probability measure on $\mathbb{R}$. Moreover, as a property of the DP, if $P \sim D P(\alpha)$, for any measurable partition of $\mathbb{R} B=\left\{B_{1}, \ldots, B_{m}\right\}$, we obtain $P\left(B_{1}\right), \ldots, P\left(B_{m}\right) \sim \operatorname{Dir}\left(\alpha\left(B_{1}\right), \ldots, \alpha\left(B_{m}\right)\right)$.

This procedure could be thought as if we started with a distribution of the probability of an algorithm outperforming another algorithm. Then, using the observations we change this distribution: so that while before it was a wider distribution with no certainty regarding the results, now it is closer to the observations made. 


\subsection{Bayesian Sign and Signed-Rank Tests}

This test is presented by Benavoli et al. [47] as a Bayesian version of sign test. We consider a DP to be the prior distribution of the scalar variable $z$. The DP is determined by the prior strength parameter $s>0$ and the prior mean $\alpha^{*}=\frac{\alpha}{s}$, which is a probability measure for DPs. We usually use the measure $\delta_{z_{0}}$, i. e. a Dirac's delta centered on the pseudo-observation $z_{0}$. Then the posterior probability density function of the difference between algorithms $Z$ follows the expression [8]:

$$
P(z)=w_{0} \delta_{z_{0}}(z)+\sum_{j=1}^{n} w_{j} \delta_{z_{j}}(z),
$$

where $\left(w_{0}, w_{1}, \ldots, w_{n}\right) \sim \operatorname{Dir}(s, 1, \ldots, 1)$, that is a combination of Dirac delta functions centered on the observations with Dirichlet distributed weights. In the sign test, we compute $\theta_{l}, \theta_{e}$ and $\theta_{r}$, the probabilities of the mean difference between algorithms being in the intervals $(-\infty,-r),[-r, r]$ or $(r,+\infty)$ respectively, where $r$ is the limit of the region of practical equivalence (rope) as a linear combination of the observations with the weights $w_{i}$. Then,

$$
\theta_{l}, \theta_{e}, \theta_{r} \sim \operatorname{Dir}\left(n_{l}+s I_{(-\infty,-r)}\left(z_{0}\right), n_{e}+s I_{[-r, r]}\left(z_{0}\right), n_{r}+s I_{(r,+\infty)}\left(z_{0}\right)\right),
$$

where $n_{l}, n_{e}, n_{r}$ are the number of observations that fall in each interval.

For a version of signed rank test the computation of the probabilities $\theta_{l}, \theta_{e}, \theta_{r}$ is similar to that of the sign test, although it involves pairs of observations in the modification of the probabilities. This time there is not a simple distribution for the probabilities, although we can estimate it using Monte Carlo sampling $\left(w_{0}, w_{1}, \ldots, w_{n}\right) \sim \operatorname{Dir}(s, 1, \ldots, 1)$.

\subsection{Imprecise Dirichlet Process}

In the Bayesian paradigm we must fix the prior strength $s$ and the prior measure $\alpha^{*}$ according to the available prior information. If we do not have any information, we should specify a non-informative Dirichlet Process. In our problem we could use the limiting DP when $s \rightarrow 0$, but it brings out numerical problems like instability in the inversion of Bayesian Friedman covariance matrix. A solution then is assuming $s>0$ and $\alpha^{*}=\delta_{X_{1}=\cdots=X_{m}}$, so we get $\mathcal{E}\left[E\left[X_{j}-X_{i}\right]\right]=0$ for each pair $i, j$ and $\mathcal{E}\left[E\left[R_{j}\right]\right]=m(m-1) / 2$. Then we are presuming that all the algorithms' performances are equal, which is not non-informative. The alternative proposed in [47] is to use a prior near-ignorance DP (IDP). This involves fixing $s>0$ and letting $\alpha^{*}$ vary in the set of all probability measures, i. e. considering all the possible prior ranks. Posterior inference is then computed taking $\alpha^{*}$ into account, obtaining lower and upper bounds for the hypothesis probability.

\subsection{Bayesian Friedman Test}

Bayesian sign test is generalised for the comparison among $m \geq 3$ algorithms by the Bayesian Friedman test [51]. In this section $\gamma$ represents the level of 
significance, as $\alpha$ denotes the measure. We consider the function $R\left(X_{i}\right)=$ $\sum_{i \neq k=1} I_{\left\{X_{i}>X_{k}\right\}}+1$, the $i$-th rank. The main goal here is to test if the point $\mu_{0}=[(m+1) / 2, \ldots,(m+1) / 2]$, i. e. the point of null hypothesis where all the algorithms have the same rank, is in $(1-\gamma) \% S C R\left(E\left[R\left(X_{1}\right), \ldots, R\left(X_{m}\right)\right]\right)$, where the $S C R$ is the symmetric credible region for $E\left[R\left(X_{1}\right), \ldots, R\left(X_{m}\right)\right]$. If the inclusion does not happen, there is a difference between algorithms with probability $1-\gamma$.

For a large number of $n$ observations, we can suppose that the mean and the covariance matrix tends to the sample mean $\mu$ and covariance $\Sigma$. Then, we define $\left.\rho=\mathrm{F}_{\text {inv }}(1-\gamma, m-1, n-m+1) \frac{(n-1)(m-1)}{n-m+1}\right\}$, where $\mathrm{F}_{\text {inv }}$ is the inverse of the $\mathrm{F}$ distribution. So we assume that $\mu_{0} \in(1-\gamma) \% S C R$ if

$$
\left.\left(\mu-\mu_{0}\right)^{T} \Sigma^{-1}\left(\mu-\mu_{0}\right)\right|_{m-1} \leq \rho,
$$

where the notation $\left.\right|_{m-1}$ means that we take the first $m-1$ components. For a small number of observations, we can compute the $S C R$ by sampling from the posterior DP, resulting in the linear combination of the weights and the Dirac delta functions centered in the observations $P=w_{0} \delta_{\mathbf{X}_{0}}+\sum_{j=1}^{n} w_{j} \delta_{\mathbf{X}_{j}}$, where $\mathbf{X}_{0}$ is the pseudo-observation.

\section{Multiple Measures Tests}

A relevant concern in the optimisation community is the comparison between multi-objective algorithms. This issue can be addressed by considering a weighted sum of the scores of the different objectives or by calculating the Pareto frontier, selecting the algorithms that are not worse than others in all the criteria or objectives. The statistical approach for this scenario consists in the examination of the relation between two studied elements (algorithms in our case) through multiple observations (benchmarks) and measuring different properties. As in the tests oriented to the comparison of single-objective optimisation algorithms, there exist statistical tests from the different paradigms that address this issue:

\subsection{Hotelling's $T^{2}$}

The parametric approach to the comparison of multivariate groups is the Hotelling's $T^{2}$ statistics [77. For this test, we would start with two matrices, gathering the results of two algorithms in $m$ measures. This test assumes that the difference from the samples comes from a multivariate Gaussian distribution $\mathcal{N}_{m}(\mu, \boldsymbol{\Sigma})$ and $\mu$ and $\boldsymbol{\Sigma}$ are unknown. This assumption can be checked with the generalised version of Shapiro-Wilk's test [78. We have not found any proposal of the use of the Hotelling's $T^{2}$ test to the comparison of algorithms, what can be motivated by the mentioned assumption required. Then, the null hypothesis is that the mean of the $m$ measures is the same for the two matrices. This means 
that if the null hypothesis is rejected, there is at least a measure where the algorithms obtain different values.

However, this test has some drawbacks, as the prerequisite of the normality of the results. Besides, in multiple objective optimisation field, the researchers are interested in finding Pareto optimal solutions, and it is not enough knowing that there are differences between the compared algorithms.

\subsection{Non-Parametric Multiple Measures Test}

A relevant proposal to multi-objective algorithms comparison comes from de Campos and Benavoli [79. In this study, they propose a new test for the comparison of the results of two algorithms in multiple problems and measures. They make a non-parametric proposal for the comparison of two algorithms and its Bayesian version.

In this comparison, we have two matrices with the results of two algorithms in $n$ rows representing the problems or benchmark functions measured in $m$ quality criteria or objectives. Then, let $M_{1}, \ldots, M_{m}$ be a set of $m$ performance measures, and, in the comparison of the algorithms $A$ and $B$, we call a dominance statement $D^{(B, A)}=[\succ, \prec, \ldots, \prec]$, where the comparison $\succ$ in the $i$-th entry of $D^{(B, A)}$ means that the algorithm $B$ is better than $A$ for $M_{i}$. Then we want to decide which (from $2^{n}$ possibilities) $D^{(B, A)}$ is the most appropriate vector given the results matrices from each algorithm.

We denote $\theta=\left[\theta_{0}, \ldots, \theta_{2^{m-1}}\right]$ the set of probabilities for each possible dominance statement and $i^{*}$ the index of the most observed configuration. Then, the null hypothesis $\mathcal{H}_{0}$ is defined as $\theta_{i^{*}} \leq \max \left(\theta \backslash \theta_{i^{*}}\right)$, i. e. rejecting the null hypothesis would mean rejecting the fact that the probability assigned to the most observed configuration is less or equal to the second greatest probability. The computation of the statistic is detailed in [79.

\subsection{Bayesian Multiple Measures Test}

The Bayesian version of the Multiple Measures Test follows a Bayesian estimation approach and estimates the posterior probability of the vector of probabilities $\theta$. A Dirichlet distribution is considered to be the prior distribution. Then the weights are updated according to the observations. The posterior probabilities of the dominance statements are computed by Monte Carlo sampling from the space of $\theta$ and counting the fraction of times for each $i$ that $\theta_{i}$ is the maximum of $\theta$.

\section{Experimental Framework}

In this section we describe the framework that will be used in the experiments in Section 8. This way the use of the previously defined test in the scenario of a statistical comparison of the CEC'17 Special Session and Competition on Single Objective Real Parameter Numerical Optimisation can be illustrated [13. 
The results have been obtained from the organiser's GitHub repository ${ }^{2}$. The mean final results for the LSHADE-cnEpSin algorithm, whose results have not been correctly included in the organisation data, have been extracted from the original paper.

\subsection{Benchmarks Functions}

The competition goal is finding the minimum of the test functions $f(\mathbf{x})$, where $\mathbf{x} \in \mathbb{R}^{D}, D \in\{10,30,50,100\}$. All the benchmark functions are shifted to a global optimum $\mathbf{o}$ and scalable and rotated according to $M_{i}$ a rotation matrix. The search range is $[-100,100]^{D}$ for all functions. Below we have included a simple summary of the test functions:

- Unimodal functions:

- Bent Cigar Function

- Sum of Different Power Function. The results of this function have been discarded in the experiments because they presented some unstable behavior for the same algorithm presented in different languages.

- Zakharov Function

- Simple Multimodal Functions:

- Disembark Function

- Expanded Scaffer's F6 Function

- Lunacek Bi-Rastrigin Function

- Non-Continuous Rastrigin's Function

- Levy Function

- Schwefel's Function

- Ten Hybrid Functions formed as the sum of different basic functions.

- Ten Composition Functions formed as a weighted sum of basic functions plus a bias according to which component optimum is the global one.

\subsection{Contestant Algorithms}

The contestant algorithms are briefly described in the following list, and are ordered according to the ranking obtained in the competition. A short-name has been assigned to each algorithm in order to present clearer tables and plots.

1. EBOwithCMAR (EBO) [80]: Effective Butterfly Optimiser with a new phase which uses Covariace Matrix (CMAR).

\footnotetext{
2 https://github.com/P-N-Suganthan/CEC2017-BoundContrained
} 
2. jSO [81]: Improved variant of iL-SHADE algorithm based on a new weighted version of mutation strategy.

3. LSHADE-cnEpSin (LSCNE) 82]: New version of LSHADE-EpSin that uses an ensemble of sinusoidal approaches based on the current adaptation and a modification of the crossover operator with a covariance matrix.

4. LSHADE-SPACMA (LSSPA) 83]: Hybrid version of a proposed algorithm LSHADE-SPA and CMA-ES.

5. DES [84]: An evolutionary algorithm that generates new individuals using a non-elitist truncation selection and an enriched differential mutation.

6. MM-OED (MM) 85]: Multi-method based evolutionary algorithm with orthogonal experiment design (OED) and factor analysis to select the best strategies and crossover operators.

7. IDE-bestNsize (IDEN) [86]: Variant of individual-dependent differential evolution with a new mutation strategy in the last phase.

8. RB-IPOP-CMA-ES (RBI) [87]: New version of IPOP-CMA-ES with a restart trigger according to the midpoint fitness.

9. MOS (MOS11, MOS12, MOS13) [88]: Three large-scale global optimiser used in these scenarios.

10. PPSO [89]: Self tuning Particle Swarm Optimisation relying on Fuzzy Logic.

11. DYYPO [90]: Version of Yin-Yang Pair Optimisation that converts a static archive updating interval into a dynamic one.

12. TLBO-FL (TFL) [91]: Variant to the Teaching Learning Based Optimisation algorithm that includes focused learning of students.

\section{Experiments and Results}

In this section we perform the previously described tests on the competition results in order to provide clear examples of their use.

\section{Setup considerations}

- Here we use the self-developed shiny application shinytests 3 , which makes use of our R package rNPBST for the analysis of the results of the competition. The associated blocks of code and scripts are available as a vignette in the rNPBST packag $\AA^{4}$

3 https://github.com/JacintoCC/shinytests

4 https://jacintocc.github.io/rNPBST/articles/StatisticalAnalysis.html 
- We will mainly use two data sets, one with all the results (all iterations of the execution of all the algorithms in all benchmark functions for all dimensions), cec17. extended.final, and the mean data set (which aggregates the results among the runs), cec17.final.

- In most of the pairwise comparisons we have involved EBOwithCMAR and jSO algorithms, as they are the best-classified algorithms in the competition.

- Table 3 shows the mean among different runs of the results obtained at the end of all of the steps of each algorithm on each benchmark function for the 10 dimension scenario.

- The tables included in this section are obtained with the function AdjustFormatTable of the package used, which is helpful to highlight the rejected hypotheses by emboldening the associated $p$-values.

\subsection{Parametric Analysis}

As we have described before, traditionally the statistical tests applied to the comparison of different algorithms belonged to the parametric family of tests. We start the statistical analysis of the results with these kinds of tests and the study of the prerequisites in order to use them safely.

The traditional parametric test used in the context of a comparison of multiple algorithms over multiple problems (benchmarks) is the ANOVA test, as we have seen in subsection 3.1. This test makes some assumptions that should be checked before it is performed:

1. The distribution of the results for each algorithm among different benchmarks follows a Gaussian distribution.

2. The standard deviation of results among groups is equal.

In Table 4 we gather the $p$-values associated with the normality of each group of mean results for an algorithm in a dimension scenario. All the null hypotheses are rejected because the $p$-values are less than 0.05 , which means that we reject that the distribution of the mean results for each benchmark function follow a normal distribution. This conclusion could be expected because of the different difficulty of the benchmark functions in higher dimension scenarios. This is marked with boldface in subsequent tables.

In some circumstances like the Multi-Objective Optimisation we need to include different measures in the comparison. We will now consider the results of the different dimensions as if they were different measures in the same benchmark function. Then, in order to perform the Hotelling's $T^{2}$ test we first check the normality of the population with the multivariate generalisation of Shapiro-Wilk's test. Table 5 shows that the normality hypothesis is rejected for every algorithm. Therefore, we stop the parametric analysis of the results here because the assumptions of parametric tests are not satisfied. 


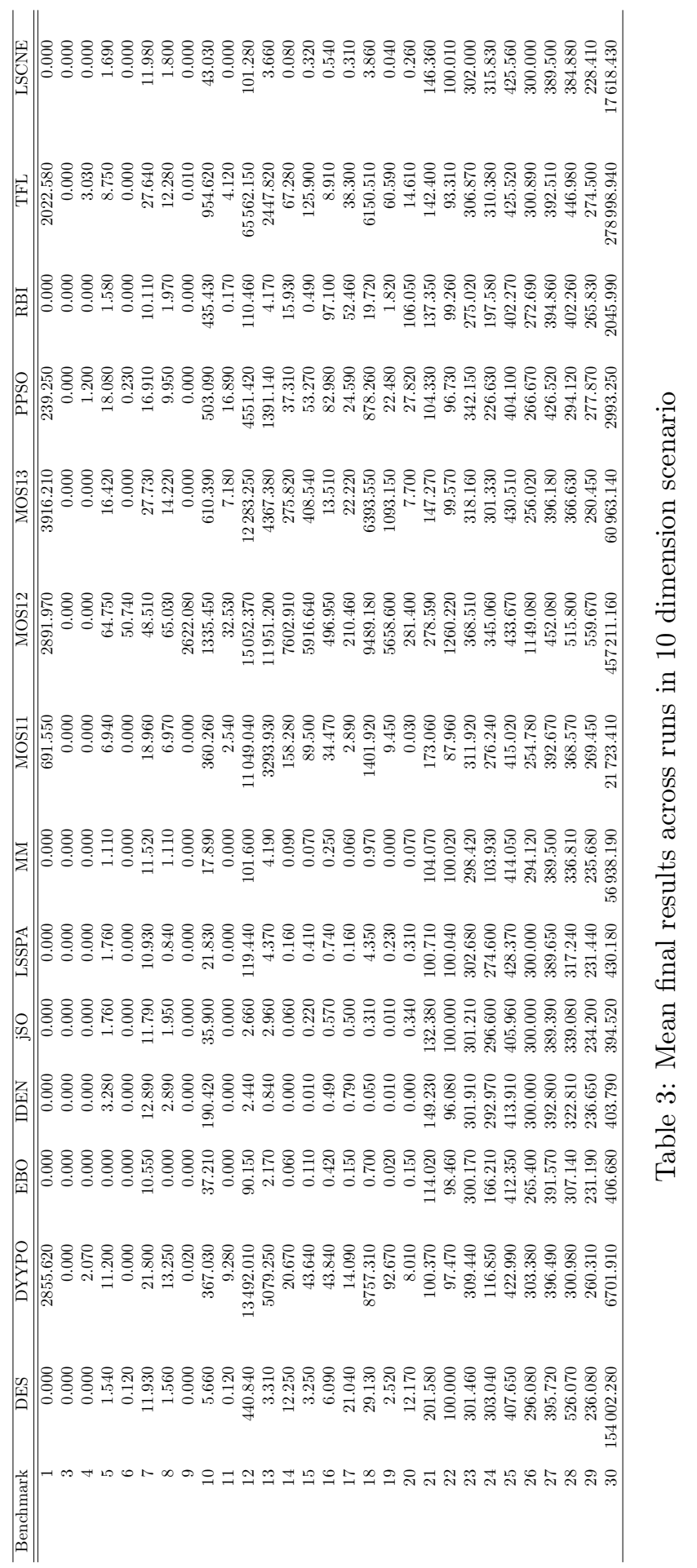




\begin{tabular}{|c|c|c|c|c|}
\hline Algorithm & Dim. 10 & Dim. 30 & Dim. 50 & Dim. 100 \\
\hline DES & $1.40 \cdot 10^{-11}$ & $6.37 \cdot 10^{-08}$ & $10^{-11}$ & $3.85 \cdot 10^{-08}$ \\
\hline DYYPO & $7.62 \cdot 10^{-09}$ & $2.35 \cdot 10^{-11}$ & $3.67 \cdot 10^{-11}$ & $1.68 \cdot 10^{-11}$ \\
\hline EBO & $2.88 \cdot 10^{-06}$ & $1.22 \cdot 10^{-07}$ & $1.39 \cdot 10^{-11}$ & $2.71 \cdot 10^{-08}$ \\
\hline IDEN & $3.15 \cdot 10^{-06}$ & $4.24 \cdot 10^{-08}$ & $1.59 \cdot 10^{-11}$ & $1.21 \cdot 10^{-10}$ \\
\hline jSO & $1.28 \cdot 10^{-06}$ & $1.42 \cdot 10^{-07}$ & $1.40 \cdot 10^{-11}$ & $9.00 \cdot 10^{-09}$ \\
\hline LSSPA & $2.84 \cdot 10^{-06}$ & $3.76 \cdot 10^{-07}$ & $1.39 \cdot 10^{-11}$ & $3.71 \cdot 10^{-08}$ \\
\hline MM & $1.51 \cdot 10^{-11}$ & $1.68 \cdot 10^{-07}$ & $1.40 \cdot 10^{-11}$ & $7.61 \cdot 10^{-07}$ \\
\hline MOS11 & $3.38 \cdot 10^{-10}$ & $3.75 \cdot 10^{-10}$ & $1.51 \cdot 10^{-10}$ & $1.89 \cdot 10^{-09}$ \\
\hline MOS12 & $2.16 \cdot 10^{-11}$ & $2.42 \cdot 10^{-08}$ & $3.64 \cdot 10^{-11}$ & $2.79 \cdot 10^{-10}$ \\
\hline MOS13 & $1.09 \cdot 10^{-10}$ & $2.82 \cdot 10^{-09}$ & $3.03 \cdot 10^{-11}$ & $9.56 \cdot 10^{-11}$ \\
\hline PPSO & $7.57 \cdot 10^{-09}$ & $1.77 \cdot 10^{-10}$ & $3.32 \cdot 10^{-10}$ & $4.49 \cdot 10^{-11}$ \\
\hline RBI & $4.75 \cdot 10^{-09}$ & $2.12 \cdot 10^{-07}$ & $5.75 \cdot 10^{-11}$ & $2.07 \cdot 10^{-10}$ \\
\hline TFL & $4.47 \cdot 10^{-11}$ & $7.50 \cdot 10^{-11}$ & $2.41 \cdot 10^{-09}$ & $1.58 \cdot 10^{-11}$ \\
\hline LSCNE & $2.17 \cdot 10^{-11}$ & $2.20 \cdot 10^{-07}$ & $1.39 \cdot 10^{-11}$ & $1.95 \cdot 10^{-08}$ \\
\hline
\end{tabular}

Table 4: $p$-values for Shapiro tests for the normality of the mean results

\subsection{Non-parametric Tests}

In this subsection we perform the most popular tests in the field of the comparison of optimisation algorithms. We continue using the aggregated results across the different runs at the end of the iterations, except for the Page test for the study of the convergence.

\subsubsection{Classic tests}

Pairwise comparisons First, we perform the non-parametric pairwise comparisons with the Sign, Wilcoxon and Wilcoxon Rank-Sum tests described in subsubsection 3.2.2 for the 10 dimension scenario. The hypotheses of the equality of the medians is only rejected by the Wilcoxon Rank-Sum test, and we can see in Table 6 how for example Wilcoxon's statistics $R+$ and $R$ - are both high numbers, which means that there is no significant difference between the ranking of the observations where one algorithm outperforms the other. Then, the next step requires all the different algorithms in the competition to be involved in the comparison.

Multiple comparisons We can see in Table 7 how the tests that involve multiple algorithms, described in subsubsection 3.2.3 reject the null hypotheses, that is, the equivalence of the medians of the results of the different benchmarks. We must keep in mind that a comparison between thirteen algorithms is not the recommended procedure if we want to compare our proposal. We should only include the state-of-the-art algorithms in the comparison, because the inclusion of an algorithm with lower performance could lead to the rejection of the null hypothesis, not due to the differences between our algorithm and the comparison 


\begin{tabular}{lr}
\hline Algorithm & p-value \\
\hline DES & $\mathbf{5 . 0 8} \cdot \mathbf{1 0}^{-\mathbf{2 1}}$ \\
DYYPO & $\mathbf{7 . 6 7} \cdot \mathbf{1 0}^{-\mathbf{3 1}}$ \\
EBO & $\mathbf{3 . 5 7} \cdot \mathbf{1 0}^{-\mathbf{2 3}}$ \\
IDEN & $\mathbf{6 . 5 4} \cdot \mathbf{1 0}^{-\mathbf{2 6}}$ \\
jSO & $\mathbf{3 . 8 2} \cdot \mathbf{1 0}^{-\mathbf{2 4}}$ \\
LSSPA & $\mathbf{4 . 7 2} \cdot \mathbf{1 0}^{-\mathbf{2 3}}$ \\
MM & $\mathbf{2 . 6 4} \cdot \mathbf{1 0}^{-\mathbf{2 1}}$ \\
MOS11 & $\mathbf{4 . 4 8} \cdot \mathbf{1 0}^{-27}$ \\
MOS12 & $\mathbf{1 . 8 4} \cdot \mathbf{1 0}^{-\mathbf{2 6}}$ \\
MOS13 & $\mathbf{7 . 5 0} \cdot \mathbf{1 0}^{-\mathbf{3 0}}$ \\
PPSO & $\mathbf{1 . 3 8} \cdot \mathbf{1 0}^{-\mathbf{2 9}}$ \\
RBI & $\mathbf{2 . 2 1} \cdot \mathbf{1 0}^{-\mathbf{2 4}}$ \\
TFL & $\mathbf{5 . 0 3} \cdot \mathbf{1 0}^{-\mathbf{2 9}}$ \\
LSCNE & $\mathbf{3 . 6 1} \cdot \mathbf{1 0}^{-\mathbf{2 3}}$ \\
\hline
\end{tabular}

Table 5: $p$-values for Multivariate Shapiro tests

\begin{tabular}{|c|c|c|c|}
\hline Test & Binomial Sign & Wilcoxon & Wilcoxon SR \\
\hline p-value & 0.211 & 0.693 & 0.00035 \\
\hline \multirow{2}{*}{ Statistics } & 8 & $\mathrm{R}+\quad 151.5$ & WRank 627 \\
\hline & K2 & 283.5 & \\
\hline
\end{tabular}

Table 6: Non-parametric pairwise comparison between EBO and jSO for 10 dimensional problems

group, but because of the differences between this dummy algorithm and the others.

\subsubsection{Post-hoc tests}

Then, we proceed to perform the post-hoc tests (described in subsection 3.3) in order to determine the location of the differences between these algorithms. We use the modification of the classic non-parametric tests to obtain the $p$-value associated with each hypothesis, although we should adjust these $p$-values with a post-hoc procedure.

Control algorithm To illustrate this, we first suppose that we are in a One versus all scenario where we are presenting our algorithm (we will use EBOwithCMAR, the winner of the CEC'17 competition). The possible approach here, as in the rest of the analysis is:

\footnotetext{
${ }^{5}$ In this and following tables, the precision used to round a number to zero is 2.220446 . $10^{-16}$.
} 


\begin{tabular}{|c|c|c|c|c|}
\hline Test & Dim. 10 & Dim. 30 & Dim. 50 & Dim. 100 \\
\hline Friedman & $9.91 \cdot 10^{-11}$ & $1.12 \cdot 10^{-10}$ & $1.08 \cdot 10^{-10}$ & $1.33 \cdot 10^{-10}$ \\
\hline Friedman AR & $0.00 \cdot 10^{+00}$ & $0.00 \cdot 10^{+00}$ & $0.00 \cdot 10^{+00}$ & $0.00 \cdot 10^{+00}$ \\
\hline Iman-Davenport & $0.00 \cdot 10^{+00}$ & $0.00 \cdot 10^{+00}$ & $0.00 \cdot 10^{+00}$ & $0.00 \cdot 10^{+00}$ \\
\hline Quade & $7.91 \cdot 10^{-37}$ & $6.87 \cdot 10^{-62}$ & $1.27 \cdot 10^{-57}$ & $2.52 \cdot 10^{-61}$ \\
\hline
\end{tabular}

Table 7: $p$-values for Non-Parametric tests for the results in the different scenarios $\$^{5}$

\begin{tabular}{|c|c|c|c|}
\hline Algorithm & Friedman & FriedmanAR & Quade \\
\hline DES & $4.26 \cdot 10^{-03}$ & $4.26 \cdot 10^{-03}$ & $8.93 \cdot 10^{-01}$ \\
\hline DYYPO & $0.00 \cdot 10^{+00}$ & $0.00 \cdot 10^{+00}$ & $2.94 \cdot 10^{-05}$ \\
\hline IDEN & $3.61 \cdot 10^{-07}$ & $3.61 \cdot 10^{-07}$ & $3.71 \cdot 10^{-01}$ \\
\hline jSO & $2.85 \cdot 10^{-01}$ & $2.85 \cdot 10^{-01}$ & $9.83 \cdot 10^{-01}$ \\
\hline LSSPA & $2.85 \cdot 10^{-01}$ & $2.85 \cdot 10^{-01}$ & $9.52 \cdot 10^{-01}$ \\
\hline MM & $5.05 \cdot 10^{-02}$ & $5.05 \cdot 10^{-02}$ & $8.93 \cdot 10^{-01}$ \\
\hline MOS11 & $0.00 \cdot 10^{+00}$ & $0.00 \cdot 10^{+00}$ & $1.62 \cdot 10^{-04}$ \\
\hline MOS12 & $0.00 \cdot 10^{+00}$ & $0.00 \cdot 10^{+00}$ & $1.45 \cdot 10^{-06}$ \\
\hline MOS13 & $0.00 \cdot 10^{+00}$ & $0.00 \cdot 10^{+00}$ & $8.00 \cdot 10^{-05}$ \\
\hline PPSO & $0.00 \cdot 10^{+00}$ & $0.00 \cdot 10^{+00}$ & $1.62 \cdot 10^{-04}$ \\
\hline RBI & $2.97 \cdot 10^{-05}$ & $2.97 \cdot 10^{-05}$ & $3.97 \cdot 10^{-01}$ \\
\hline TFL & $0.00 \cdot 10^{+00}$ & $0.00 \cdot 10^{+00}$ & $4.04 \cdot 10^{-06}$ \\
\hline LSCNE & $2.85 \cdot 10^{-01}$ & $2.85 \cdot 10^{-01}$ & $9.83 \cdot 10^{-01}$ \\
\hline
\end{tabular}

Table 8: Post-Hoc Non-Parametric tests with control algorithm

- Considering all the results in the different dimensions as if they were different function or benchmarks, we would only obtain a single $p$-value for the comparison between EBO-CMAR with each contestant algorithm. The adjusted $p$-values are shown in Table 8 for the Friedman, Friedman Aligned-Rank and Quade test. Here we see that there is not much difference between the different tests and that differences are found in the comparison with DYYPO, IDEN, MOS, PPSO, RBI and TFL, although the sign of these differences need to be checked in the raw data.

- If we wanted to show that the differences between the algorithms also persist in each group of results obtained across the different dimensions, we should perform these tests repeatedly and apply the appropiate posthoc procedure later. In Table 9 we show the adjusted $p$-values. 


\begin{tabular}{|c|c|c|c|c|}
\hline & Dim 10 & Dim 30 & Dim 50 & Dim 100 \\
\hline DES & $8.35 \cdot 10^{-04}$ & $1.24 \cdot 10^{-01}$ & $9.95 \cdot 10^{-01}$ & $9.85 \cdot 10^{-01}$ \\
\hline DYYPO & $3.67 \cdot 10^{-07}$ & $2.15 \cdot 10^{-12}$ & $1.44 \cdot 10^{-09}$ & $3.82 \cdot 10^{-09}$ \\
\hline IDEN & $9.67 \cdot 10^{-01}$ & $1.19 \cdot 10^{-01}$ & $1.49 \cdot 10^{-02}$ & $1.80 \cdot 10^{-02}$ \\
\hline jSO & $9.86 \cdot 10^{-01}$ & $9.96 \cdot 10^{-01}$ & $9.96 \cdot 10^{-01}$ & $9.96 \cdot 10^{-01}$ \\
\hline LSSPA & $6.35 \cdot 10^{-01}$ & $8.96 \cdot 10^{-01}$ & $9.96 \cdot 10^{-01}$ & $9.96 \cdot 10^{-01}$ \\
\hline MM & $9.96 \cdot 10^{-01}$ & $8.61 \cdot 10^{-01}$ & $6.35 \cdot 10^{-01}$ & $9.96 \cdot 10^{-01}$ \\
\hline MOS11 & $1.79 \cdot 10^{-04}$ & $4.94 \cdot 10^{-09}$ & $3.49 \cdot 10^{-08}$ & $2.70 \cdot 10^{-05}$ \\
\hline MOS12 & $0.00 \cdot 10^{+00}$ & $0.00 \cdot 10^{+00}$ & $4.44 \cdot 10^{-13}$ & $2.10 \cdot 10^{-09}$ \\
\hline MOS13 & $3.82 \cdot 10^{-09}$ & $6.17 \cdot 10^{-12}$ & $4.47 \cdot 10^{-11}$ & $1.80 \cdot 10^{-06}$ \\
\hline PPSO & $2.19 \cdot 10^{-05}$ & $4.53 \cdot 10^{-10}$ & $3.64 \cdot 10^{-11}$ & $1.47 \cdot 10^{-08}$ \\
\hline RBI & $3.74 \cdot 10^{-02}$ & $6.65 \cdot 10^{-02}$ & $5.98 \cdot 10^{-01}$ & $9.92 \cdot 10^{-01}$ \\
\hline TFL & $1.44 \cdot 10^{-09}$ & $6.80 \cdot 10^{-12}$ & $2.96 \cdot 10^{-11}$ & $1.51 \cdot 10^{-11}$ \\
\hline LSCNE & $3.85 \cdot 10^{-01}$ & $9.95 \cdot 10^{-01}$ & $9.96 \cdot 10^{-01}$ & $9.96 \cdot 10^{-01}$ \\
\hline
\end{tabular}

Table 9: Results grouped by dimension. Friedman test + Holland adjust using EBO-CMAR as control algorithm.

$n$ versus $n$ scenario In the scenario of the statistical analysis of the results obtained during a competition, we do not focus on the comparison of the results of a single algorithm, rather we would make all the posible pairs, and therfore we would not use the control algorithm.

The results of the $n \times n$ comparison using a Friedman test and a Post-Hoc Holland adjust of the $p$-values is shown in Tables 10 and 11 . In these tables we can see that there is not a single algorithm whose equivalence with the rest of the algorithms is discarded (in the 10 dimension scenario). However, for a multiple comparison with a high number of algorithms, like in the competition used as example, the adjustment makes finding differences between the algorithms more difficult. If we observe the results of the best classified algorithms in the competition, like jSO and EBO, we see that there are significant differences with algorithms like MOS, PPSO or RBI but this difference is not significant for LSHADE variants or MM.

\begin{tabular}{|c|c|c|c|c|c|c|c|}
\hline & DES & DYYPO & EBO & IDEN & jSO & LSSPA & MM \\
\hline DES & & $9.99 \cdot 10^{-01}$ & $3.69 \cdot 10^{-03}$ & $2.94 \cdot 10^{-01}$ & $1.77 \cdot 10^{-01}$ & $8.44 \cdot 10^{-01}$ & $5.48 \cdot 10^{-02}$ \\
\hline DYYPO & $9.99 \cdot 10^{-01}$ & & $1.78 \cdot 10^{-06}$ & $1.20 \cdot 10^{-03}$ & $5.12 \cdot 10^{-04}$ & $1.82 \cdot 10^{-02}$ & $7.67 \cdot 10^{-05}$ \\
\hline EBO & $3.69 \cdot 10^{-03}$ & $1.78 \cdot 10^{-06}$ & & $1.00 \cdot 10^{+00}$ & $1.00 \cdot 10^{+00}$ & $9.70 \cdot 10^{-01}$ & $1.00 \cdot 10^{+00}$ \\
\hline IDEN & $2.94 \cdot 10^{-01}$ & $1.20 \cdot 10^{-03}$ & $1.00 \cdot 10^{+00}$ & & $1.00 \cdot 10^{+00}$ & $1.00 \cdot 10^{+00}$ & $1.00 \cdot 10^{+00}$ \\
\hline jSO & $1.77 \cdot 10^{-01}$ & $5.12 \cdot 10^{-04}$ & $1.00 \cdot 10^{+00}$ & $1.00 \cdot 10^{+00}$ & & $1.00 \cdot 10^{+00}$ & $1.00 \cdot 10^{+00}$ \\
\hline LSSPA & $8.44 \cdot 10^{-01}$ & $1.82 \cdot 10^{-02}$ & $9.70 \cdot 10^{-01}$ & $1.00 \cdot 10^{+00}$ & $1.00 \cdot 10^{+00}$ & & $1.00 \cdot 10^{+00}$ \\
\hline MM & $5.48 \cdot 10^{-02}$ & $7.67 \cdot 10^{-05}$ & $1.00 \cdot 10^{+00}$ & $1.00 \cdot 10^{+00}$ & $1.00 \cdot 10^{+00}$ & $1.00 \cdot 10^{+00}$ & \\
\hline MOS11 & $1.00 \cdot 10^{+00}$ & $1.00 \cdot 10^{+00}$ & $8.41 \cdot 10^{-04}$ & $1.12 \cdot 10^{-01}$ & $6.16 \cdot 10^{-02}$ & $5.73 \cdot 10^{-01}$ & $1.53 \cdot 10^{-02}$ \\
\hline MOS12 & $7.67 \cdot 10^{-05}$ & $5.48 \cdot 10^{-02}$ & $0.00 \cdot 10^{+00}$ & $3.91 \cdot 10^{-13}$ & $7.90 \cdot 10^{-14}$ & $6.43 \cdot 10^{-11}$ & $0.00 \cdot 10^{+00}$ \\
\hline MOS13 & $8.23 \cdot 10^{-01}$ & $1.00 \cdot 10^{+00}$ & $1.69 \cdot 10^{-08}$ & $3.12 \cdot 10^{-05}$ & $1.14 \cdot 10^{-05}$ & $7.92 \cdot 10^{-04}$ & $1.24 \cdot 10^{-06}$ \\
\hline PPSO & $1.00 \cdot 10^{+00}$ & $1.00 \cdot 10^{+00}$ & $1.03 \cdot 10^{-04}$ & $2.59 \cdot 10^{-02}$ & $1.28 \cdot 10^{-02}$ & $2.10 \cdot 10^{-01}$ & $2.55 \cdot 10^{-03}$ \\
\hline RBI & $1.00 \cdot 10^{+00}$ & $6.05 \cdot 10^{-01}$ & $1.41 \cdot 10^{-01}$ & $9.75 \cdot 10^{-01}$ & $9.22 \cdot 10^{-01}$ & $1.00 \cdot 10^{+00}$ & $6.62 \cdot 10^{-01}$ \\
\hline TFL & $6.85 \cdot 10^{-01}$ & $1.00 \cdot 10^{+00}$ & $5.99 \cdot 10^{-09}$ & $1.34 \cdot 10^{-05}$ & $4.78 \cdot 10^{-06}$ & $3.88 \cdot 10^{-04}$ & $4.90 \cdot 10^{-07}$ \\
\hline LSCNE & $9.75 \cdot 10^{-01}$ & $6.16 \cdot 10^{-02}$ & $8.28 \cdot 10^{-01}$ & $1.00 \cdot 10^{+00}$ & $1.00 \cdot 10^{+00}$ & $1.00 \cdot 10^{+00}$ & $9.98 \cdot 10^{-01}$ \\
\hline
\end{tabular}

Table 10: Results $n$ vs $n$, dimension 10. Friedman test + Holland adjust. 


\begin{tabular}{|c|c|c|c|c|c|c|c|}
\hline & MOS11 & MOS12 & MOS13 & PPSO & RBI & TFL & LSCNE \\
\hline DES & $1.00 \cdot 10^{+00}$ & $7.67 \cdot 10^{-05}$ & $8.23 \cdot 10^{-01}$ & $1.00 \cdot 10^{+00}$ & $1.00 \cdot 10^{+00}$ & $6.85 \cdot 10^{-01}$ & $9.75 \cdot 10^{-01}$ \\
\hline DYYPO & $1.00 \cdot 10^{+00}$ & $5.48 \cdot 10^{-02}$ & $1.00 \cdot 10^{+00}$ & $1.00 \cdot 10^{+00}$ & $6.05 \cdot 10^{-01}$ & $1.00 \cdot 10^{+00}$ & $6.16 \cdot 10^{-02}$ \\
\hline $\mathrm{EBO}$ & $8.41 \cdot 10^{-04}$ & $0.00 \cdot 10^{+00}$ & $1.69 \cdot 10^{-08}$ & $1.03 \cdot 10^{-04}$ & $1.41 \cdot 10^{-01}$ & $5.99 \cdot 10^{-09}$ & $8.28 \cdot 10^{-01}$ \\
\hline IDEN & $1.12 \cdot 10^{-01}$ & $3.91 \cdot 10^{-13}$ & $3.12 \cdot 10^{-05}$ & $2.59 \cdot 10^{-02}$ & $9.75 \cdot 10^{-01}$ & $1.34 \cdot 10^{-05}$ & $1.00 \cdot 10^{+00}$ \\
\hline jSO & $6.16 \cdot 10^{-02}$ & $7.90 \cdot 10^{-14}$ & $1.14 \cdot 10^{-05}$ & $1.28 \cdot 10^{-02}$ & $9.22 \cdot 10^{-01}$ & $4.78 \cdot 10^{-06}$ & $1.00 \cdot 10^{+00}$ \\
\hline LSSPA & $5.73 \cdot 10^{-01}$ & $6.43 \cdot 10^{-11}$ & $7.92 \cdot 10^{-04}$ & $2.10 \cdot 10^{-01}$ & $1.00 \cdot 10^{+00}$ & $3.88 \cdot 10^{-04}$ & $1.00 \cdot 10^{+00}$ \\
\hline MM & $1.53 \cdot 10^{-02}$ & $0.00 \cdot 10^{+00}$ & $1.24 \cdot 10^{-06}$ & $2.55 \cdot 10^{-03}$ & $6.62 \cdot 10^{-01}$ & $4.90 \cdot 10^{-07}$ & $9.98 \cdot 10^{-01}$ \\
\hline MOS11 & & $4.14 \cdot 10^{-04}$ & $9.70 \cdot 10^{-01}$ & $1.00 \cdot 10^{+00}$ & $1.00 \cdot 10^{+00}$ & $9.20 \cdot 10^{-01}$ & $8.44 \cdot 10^{-01}$ \\
\hline MOS12 & $4.14 \cdot 10^{-04}$ & & $4.42 \cdot 10^{-01}$ & $2.89 \cdot 10^{-03}$ & $2.78 \cdot 10^{-07}$ & $5.81 \cdot 10^{-01}$ & $7.71 \cdot 10^{-10}$ \\
\hline MOS13 & $9.70 \cdot 10^{-01}$ & $4.42 \cdot 10^{-01}$ & & $9.99 \cdot 10^{-01}$ & $9.71 \cdot 10^{-02}$ & $1.00 \cdot 10^{+00}$ & $3.50 \cdot 10^{-03}$ \\
\hline PPSO & $1.00 \cdot 10^{+00}$ & $2.89 \cdot 10^{-03}$ & $9.99 \cdot 10^{-01}$ & & $9.88 \cdot 10^{-01}$ & $9.97 \cdot 10^{-01}$ & $4.82 \cdot 10^{-01}$ \\
\hline RBI & $1.00 \cdot 10^{+00}$ & $2.78 \cdot 10^{-07}$ & $9.71 \cdot 10^{-02}$ & $9.88 \cdot 10^{-01}$ & & $5.92 \cdot 10^{-02}$ & $1.00 \cdot 10^{+00}$ \\
\hline TFL & $9.20 \cdot 10^{-01}$ & $5.81 \cdot 10^{-01}$ & $1.00 \cdot 10^{+00}$ & $9.97 \cdot 10^{-01}$ & $5.92 \cdot 10^{-02}$ & & $1.82 \cdot 10^{-03}$ \\
\hline LSCNE & $8.44 \cdot 10^{-01}$ & $7.71 \cdot 10^{-10}$ & $3.50 \cdot 10^{-03}$ & $4.82 \cdot 10^{-01}$ & $1.00 \cdot 10^{+00}$ & $1.82 \cdot 10^{-03}$ & \\
\hline
\end{tabular}

Table 11: Results $n$ vs $n$, dimension 10. Friedman test + Holland adjust (cont.).

The CD plot associated with the scenario of an $n$ vs. $n$ comparison is described in subsection 3.3 and performed with the Nemenyi test provides an interesting visualisation fo the significance of the observed paired differences. In Figure 2, we show the results of this comparison, where the differences between the group of the first classified algorithms whose equivalence cannot be discarded includes up to the RBI algorithm (7th classified in the 10 Dimension scenario). This plot, with several overlapped groups that contain many algorithms, shows that the differences are hard to identify in algorithms that perform similarly.

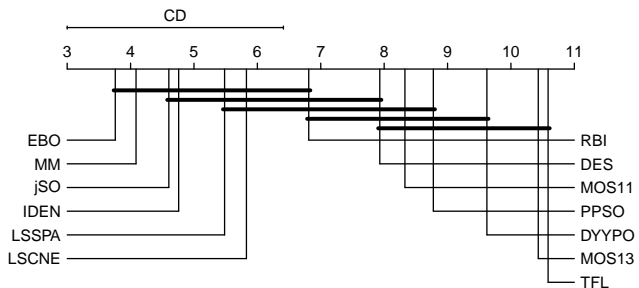

Figure 2: CD plot for 10 dimensional CEC 2017 functions

\subsubsection{Convergence test}

For the comparison of the convergence of two algorithms, we use the Page test described in subsubsection 3.2.4 with the mean result across the different runs for each benchmark function of two algorithms. These results could be equally extended using the convenient adjustments. It is relevant to note that the LSCNE algorithm (third classified) does not provide its partial results.

The results in Table 12 show the comparison of the convergence of the jSO and the LSSPA algorithms in the competition for the 10 and the comparison of the convergence of jSO and DYYPO algorithms for 100 dimension scenario. Here, the null hypothesis of the difference between LSSPA and jSO getting a positive trend cannot be rejected in the 10 dimension scenario. In the 100 


\begin{tabular}{|c|c|c|c|}
\hline \multicolumn{4}{|c|}{ Page test } \\
\hline \multicolumn{4}{|c|}{10 dimensions scenario } \\
\hline Comparison & & LSSPA-jSO & jSO-LSSPA \\
\hline statistic & $\mathrm{L}$ & 22902 & 22773 \\
\hline p.value & & 0.4253 & 0.5759 \\
\hline \multicolumn{4}{|c|}{100 dimensions scenario } \\
\hline Comparison & & jSO-DYYPO (100) & DYYPO-jSO (100) \\
\hline statistic & $\mathrm{L}$ & 25730 & 19945 \\
\hline p.value & & 0 & 1 \\
\hline
\end{tabular}

Table 12: Page test

dimension scenario, the test detects an increasing trend in the ranks of the difference between jSO and DYYPO as the null hypothesis is rejected.

\subsubsection{Confidence Intervals and Confidence Curves}

In this subsection, we show the use of confidence intervals and confidence curves in the comparison of optimisation results as mentioned in subsection 3.4. First, we must advise that these comparisons only take care of two algorithms at a time, and a post-hoc correction would be needed if the comparison involved a greater number of algorithms, as we will see in the following examples.

We perform the comparison of the final results of PPSO and jSO algorithms for the 10 dimension scenario. Results are shown in Table 13 .

As the 0 effect is not included in the non-parametric confidence interval, the null hypothesis is then rejected. The interval is very wide, so we have not much certainty the true location of the parameter. If we only had done the Wilcoxon test, we would have obtained the associated $p$-value, and the null hypothesis would also be rejected, so the difference between the medians are detected with both methods. If we look at the confidence curve, we can reject the classic null hypothesis if the interval bounded by the intersections of the horizontal line at the $\alpha$ value and the curve does not contain 0 . The confidence curve associated with the previous test is plotted in Figure 3, where we check that the null hypothesis can be rejected. The estimated difference is represented with the dotted vertical line. The width of the intersection between the curve and the dotted horizontal line indicates that there is not much certainty about the true location of the parameter, primarily in the upper bound.

\subsection{Bayesian Tests}

In this subsection we illustrate the use of the described Bayesian tests. The considerations are analogous to the ones made in the frequentist case, as the described tests use the aggregations of the runs to compare the results of the different benchmark functions, or use these runs with the drawback of obtaining a restrained statement about the results in one single problem. 


\begin{tabular}{lll}
\hline \multicolumn{3}{l}{ Wilcoxon test } \\
\hline Comparison & & \\
\hline \multirow{2}{*}{ statistic } & $\mathrm{R}+$ & 351.50 \\
& $\mathrm{R}-$ & 83.50 \\
\hline \multirow{2}{*}{ p-value } & Exact Double pvalue & $\mathbf{0 . 0 0 1 3 9 9 0}$ \\
& Asymptotic Double Tail & $\mathbf{0 . 0 0 0 0 9 9 4}$ \\
\hline \multirow{2}{*}{ Associated NP Confidence Interval } & Lower Bound & 8.557851 \\
& Upper Bound & 233.709039 \\
\hline
\end{tabular}

Table 13: Wilcoxon test and Non-Parametric Confidence Interval

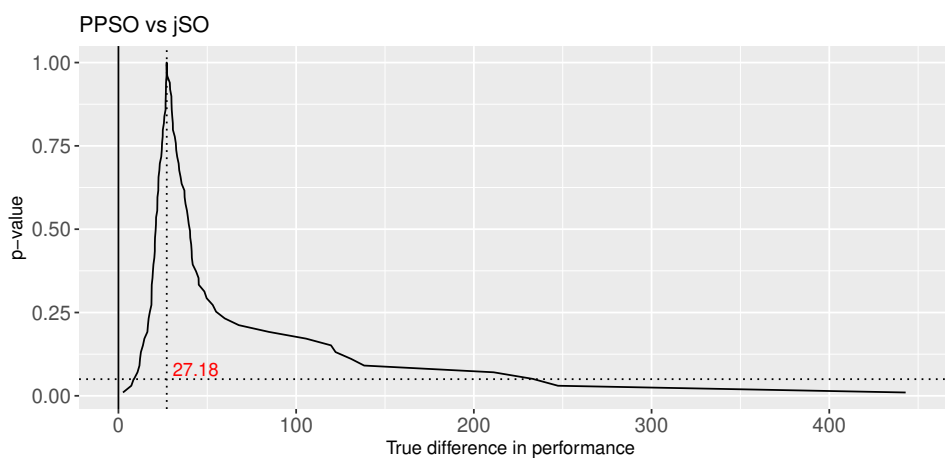

Figure 3: Confidence curve 


\subsubsection{Bayesian Friedman test}

We start with the Bayesian version of the Friedman test, mentioned in subsection 5.4. In this test we do not obtain a single $p$-value, but the accepted hypothesis. Due to the high number of contestant algorithms and the memory needed to allocate the covariance matrix of all the possible permutations, here we will perform the imprecise version of the test. The null hypothesis of the equivalence of the mean ranks for the 10 dimension scenario is rejected. The mean ranks of the algorithms are shown in Table 14.

\begin{tabular}{rr}
\hline Algorithm & Mean Rank \\
\hline DES & 7.98 \\
DYYPO & 9.62 \\
EBO & 3.55 \\
IDEN & 4.9 \\
jSO & 4.7 \\
LSSPA & 5.6 \\
MM & 4.28 \\
MOS-11 & 8.35 \\
MOS-12 & 13.32 \\
MOS-13 & 10.41 \\
PPSO & 8.81 \\
RBI & 6.01 \\
TFL & 10.58 \\
LSCNE & 5.9 \\
\hline
\end{tabular}

Table 14: Mean Rank of Bayesian Friedman Test

\subsubsection{Bayesian Sign and Signed-Rank test}

The original proposal of the use of the Bayesian Sign and Signed-Rank tests included in subsection 5.2 is the comparison of classification algorithms and the proposed rope is $[-0.01,0.01]$ for a measure in the range $[0,1]$. In the scenario of optimisation problems, we should be concerned that the possible outcomes are lower-bounded by 0 but in many functions, there is not an upper bound or the maximum is very high, so we must follow another approach. As the difference in the 100 dimension comparison is between 0 and 15000, we state that the region of practical equivalence is $[-10,10]$.

The tests compute the probability of the true location of EBO-CMAR - jSO with respect to 0 , so both tests' results shows that there is a similar probability for the three hypotheses. In the Bayesian Sign test results, the hypothesis with a greater probability is the left region (i.e. the true location is less than 0 and then jSO obtain worse results), although the results are not significant enough to state that EBO is the winner algorithm. Following the results of the Bayesian Signed-Rank test, left region is also the most probable, although this 
is not significant. Rope probability is also high, so the equivalence cannot be discarded, which means that there are several ties in the benchmark results.

We can see the posterior probability density of the parameter in Figure 4. where each point represents an estimation of the probability of the parameter which belongs to each region of interest. The vertexes of the triangle represent the points where there is probability 1 of the true location being in this region. The proportion of the location of the points is shown in Table 15. This means that we have repeatedly obtained the triplets of the probability of each region to be the true location of the difference between the two samples, and then we have plotted these triplets to obtain the posterior distribution of the parameter. If we compare these results with a paired Wilcoxon test, we see that the null hypothesis of the equivalence of the means is rejected, although there is no information about if one algorithm outperforms the other. However, using the Bayesian paradigm we can see that this is not the situation, as we cannot establish the dominance of one algorithm over the other either. Following the frequentist paradigm we could be tempted to (erroneously) establish that EBO is better, according to a single statistic and the result of the Wilcoxon test.

\begin{tabular}{lll}
\hline Wilcoxon Signed Ranks & & \\
\hline Statistic & V & 655. \\
p-value & & $\mathbf{0 . 0 0 2}$ \\
\hline Bayesian Sign & & \\
\hline \multirow{2}{*}{ Posterior probability } & jSO & 0.2777823 \\
& rope & 0.3112033 \\
& EBO & 0.4110144 \\
\hline Bayesian Signed-Rank & & \\
\hline \multirow{2}{*}{ Posterior probability } & jSO & 0.2905800 \\
& rope & 0.3409561 \\
& EBO & 0.3684639 \\
\hline
\end{tabular}

Table 15: Bayesian Sign and Signed Ranks tests
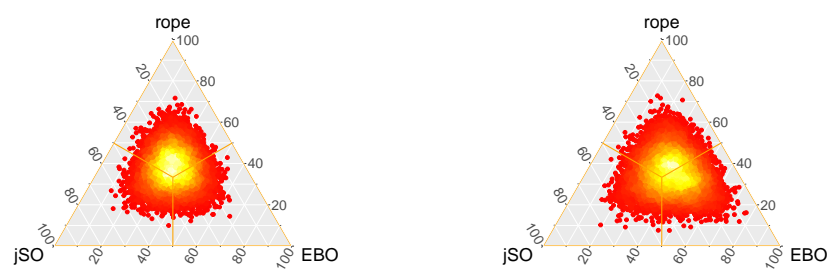

Figure 4: Bayesian Sign and Bayesian Signed-Rank tests 


\subsubsection{Imprecise Dirichlet Process Test}

The Imprecise Dirichlet Process, as we have seen in subsection 5.3, consists of a more complex idea of the previous tests although the implications of the use of the Bayesian Tests could be clearer. In this test, we try to not introduce any prior distribution, not even the prior distribution where both algorithms have the same performance, but all the possible probability measures $\alpha$, and then obtain an upper and a lower bounds for the probability in which we are interested. The input consists of the aggregated results among the different runs for all the benchmark functions for a single dimension. The other parameters of the function are the strong parameter $s$ and the pseudo-observation $c$. With these data, we obtain two bounds for the posterior probability of the first algorithm outperforming the second one, i.e. the probability of $P(X \leq Y) \geq 0.5$. These are the possible scenarios:

- Both bounds are greater than 0.95: Then we can say that the first algorithm outperforms the second algorithm with $95 \%$ probability.

- Both bounds are lower than 0.05: This is the inverse case. In this situation, the second algorithm outperforms the first algorithm with $95 \%$ probability.

- Both bounds are between 0.05 and 0.95: Then we can say that the probability of one algorithm outperforming the other is lower than the desired probability of 0.95 .

- Finally, if only one of the bounds is greater than 0.95 or lower than 0.05 , the situation is undetermined and we cannot decide.

\begin{tabular}{lcc}
\hline \multicolumn{3}{c}{ IDP - Wilcoxon test } \\
\hline \multirow{2}{*}{ Posterior Distribution } & Upper Bound & 0.591 \\
& Lower Bound & 0.452 \\
\hline
\end{tabular}

Table 16: Imprecise Dirichlet Process of Wilcoxon test

According to the results of the Bayesian Imprecise Dirichlet Process (see Table 16), the probability under the Dirichlet Process of $P(E B O \leq j S O) \geq 0.5$, that is the probability of EBO-CMAR outperforming jSO, is between 0.59 and 0.45 , so there is not a probability greater than 0.95 of EBO-CMAR outperforming jSO. These numbers represent the area under the curve of the upper and lower distributions when $P(X \leq Y) \geq 0.5$. In Figure 5 we can see both posterior distributions. 


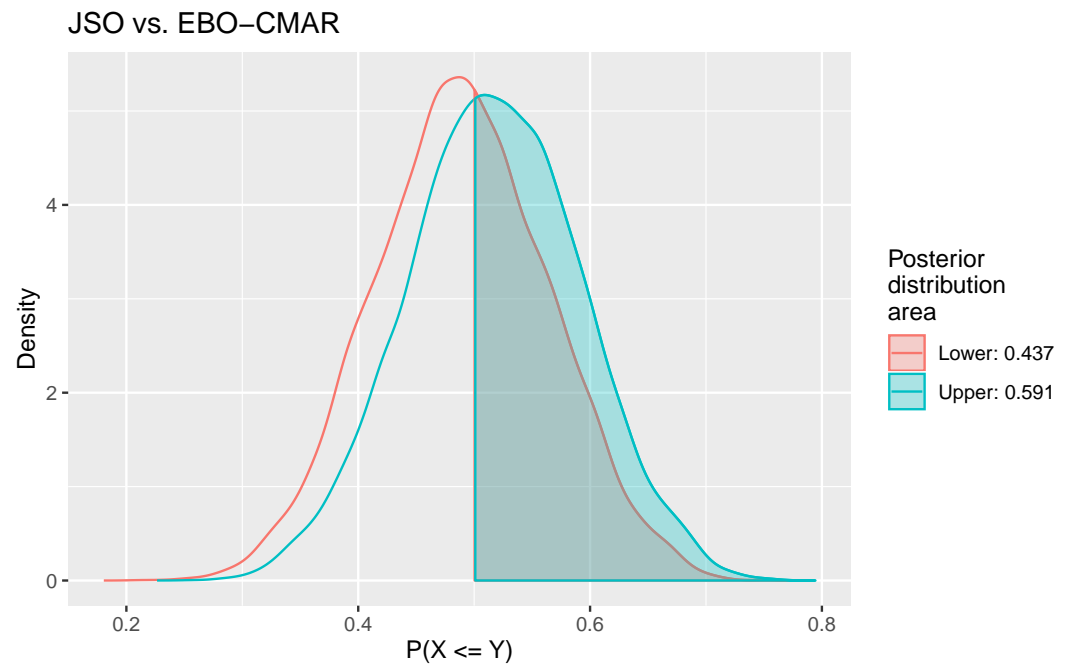

Figure 5: Imprecise Dirichlet Process - Wilcoxon test

\subsection{Multi-Objective Comparison}

In some circumstances like the Multi-Objective Optimisation, we need to include different measures in the comparison. We include in this section the illustration of the use of the tests presented in Section 6 .

\subsubsection{Multiple measures test - GLRT}

As we have mentioned in subsection 8.1 concerning the Hotelling's $T^{2}$ test, we can be interested in the simultaneous comparison of multiple measures. This is the scenario of application of the Non-Parametric Multiple Measures test, described in subsubsection 8.4.1. We select the means of the executions of the two best algorithms and reshape them into a matrix with the results of each benchmark in the rows and the different dimensions in the columns. Then we use the test to see which hypothesis of dominance is the most probable and if we can state that the probability of this dominance statement is significant. According to the results shown in Table 17, we obtain that the most observed dominance statement is the configuration $[<,<,<,<]$, it is, EBO-CMAR obtains a better result for all the dimensions. However, the associated null hypothesis which states that the mentioned configuration is no more probable than the following one, obtain a $p$-value of 0.39 , showed in Table 17, so this hypothesis cannot be rejected. The second most probable configuration is $[>,>,>,>]$ which means that EBO-CMAR obtains worse results in all the dimensions, so we cannot be certain which is the most probable situation in any scenario. It is relevant to note that the number of observations can be a real value as the weight of an observation is divided between the possible configuration when there is a tie for any measure. 


\begin{tabular}{ll}
\hline \multicolumn{2}{c}{ GLRT Multiple Measures } \\
\hline$\lambda$ & 0.6917945 \\
\hline Configuration & Number of observations \\
\hline$<<<<$ & 8.25 \\
$<<<>$ & 2.62 \\
$<<><$ & 1.25 \\
$<<>>$ & 0.12 \\
$<><<$ & 1.25 \\
$<><>$ & 0.12 \\
$<>><$ & 1.25 \\
$<>>>$ & 3.12 \\
$><<<$ & 0.75 \\
$><<>$ & 1.62 \\
$><><$ & 1.25 \\
$><>>$ & 0.12 \\
$>><<$ & 0.75 \\
$>><>$ & 0.12 \\
$>>><$ & 1.25 \\
$>>>>$ & 5.12 \\
\hline$p$-value & 0.3906 \\
\hline
\end{tabular}

Table 17: Posterior configuration probability

\subsubsection{Bayesian Multiple Measures Test}

In the Bayesian version of the Multiple Measures test the results are analogous to the frequentist version shown in the previous section. We use the same matrices with the results of each algorithm arranged by dimensions. Here we obtain that the most probable configuration is also the dominance of EBO in all the dimensions according to this test, but the posterior probability is 0.75 , as is shown in Table 18, so we cannot say that the difference with respect to the remaining configurations is determinant.

\section{Summary of results in CEC'2017}

In this section, we include a summary of the statistical analysis performed within the context of the CEC'2017.

Table 19 shows the official results of the algorithms in their scoring system and the scores computed following the indications of the report of the problem definition for the competition [13] with the available raw results of the algorithms. The Score 1 is defined using a weighted sum of the errors of the algorithms in all benchmark on different dimensions. The weights are $0.1,0.2,0.3,0.4$, with the higher weights corresponding with the higher dimension scenarios. Then, if we call $S E$ the summed error for an algorithm and 


\begin{tabular}{lc}
\hline Configuration & Probability \\
\hline$<<<<$ & 0.750 \\
$<<<>$ & 0.020 \\
$<<><$ & 0.000 \\
$<<>>$ & 0.000 \\
$<><<$ & 0.000 \\
$<><>$ & 0.000 \\
$<>><$ & 0.000 \\
$<>>>$ & 0.030 \\
$><<<$ & 0.000 \\
$><<>$ & 0.010 \\
$><><$ & 0.000 \\
$><>>$ & 0.000 \\
$>><<$ & 0.000 \\
$>><>$ & 0.000 \\
$>>><$ & 0.000 \\
$>>>>$ & 0.170 \\
\hline
\end{tabular}

Table 18: Posterior configuration probability

$S E_{\min }$ the minimum sum for an algorithm, Score 1 is defined as Score $1=$ $0.5 *\left(1-\frac{S E-S E_{\min }}{S E}\right)$. Analogously, $S$ core 2 is defined as a weighted sum of the ranks of the algorithms instead of using the error: $S$ core $2=0.5 *\left(1-\frac{S R-S R_{\min }}{S R}\right)$, where $S R$ is the weighted sum of the ranks. The difference between the official score and our computation may reside in the aggregation method for the results of the different runs or the programming language used for the computation of the scores. Different versions of the results have been used without major impact in the final ranking, which proves the robustness of the ranking and the algorithms. However, these differences do not affect the final ranking of the first classified algorithms. In the official CEC'17 summary, the results of the MOS12 algorithm are not included, so we have excluded them in the analyses of this section.

The classic statistical analysis that should be made in the context of a competition would involve a non-parametric test with post-hoc test for a $n$ versus $n$ scenario, as we do not have a preference for the results of comparison of any specific algorithm. In order to preserve the relative importance of the results in the different dimension scenarios, we show the plots of the critical difference explained in subsection 3.3 for the four scenarios.

As we can see in Figure 6, summary scores presented in Table 19 are consistent with the Critical Differences plots made with the mean values, as the first classified algorithms are also in the first positions of the graphical representation. However, the statistical tests make it possible to address the fact that there is a group of algorithms in the lead group in every dimension scenario whose associated hypothesis of equivalence cannot be discarded. These algorithms are LSSPA, DES, LSCNE, MM, jSO and EBO. Moreover, there is not a 


\begin{tabular}{|lrrr|lrrr|}
\hline \multicolumn{4}{|c|}{ Official CEC'17 Results } & \multicolumn{4}{c|}{ Score Computation } \\
\hline Algorithm & Score1 & Score2 & Score & Algorithm & Score1 & Score2 & Score \\
\hline EBO & $\mathbf{5 0 . 0 0}$ & 48.01 & $\mathbf{9 8 . 0 1}$ & EBO & $\mathbf{5 0 . 0 0}$ & $\mathbf{5 0 . 0 0}$ & $\mathbf{1 0 0 . 0 0}$ \\
jSO & 49.69 & 47.08 & 96.77 & jSO & 49.69 & 43.01 & 92.70 \\
LSCNE & 46.82 & 49.74 & 96.56 & LSCNE & 46.82 & 44.75 & 91.56 \\
LSSPA & 46.44 & $\mathbf{5 0 . 0 0}$ & 96.44 & LSSPA & 46.44 & 44.73 & 91.17 \\
DES & 45.94 & 43.20 & 89.14 & DES & 45.94 & 40.65 & 86.59 \\
MM & 45.96 & 40.12 & 86.07 & MM & 45.96 & 36.16 & 82.12 \\
IDEN & 29.85 & 27.68 & 57.53 & IDEN & 29.85 & 26.15 & 56.00 \\
RBI & 3.79 & 33.61 & 37.40 & MOS13 & 18.94 & 17.33 & 36.27 \\
MOS13 & 18.94 & 17.34 & 36.28 & RBI & 3.79 & 32.00 & 35.79 \\
MOS11 & 11.09 & 19.30 & 30.39 & MOS11 & 11.09 & 19.17 & 30.25 \\
PPSO & 3.93 & 17.36 & 21.28 & PPSO & 3.93 & 17.26 & 21.19 \\
DYYPO & 0.59 & 17.03 & 17.62 & DYYPO & 0.59 & 17.06 & 17.65 \\
TFL & 0.03 & 16.25 & 16.27 & TFL & 0.03 & 16.31 & 16.34 \\
\hline
\end{tabular}

Table 19: CEC17 Results Scores with mean results

single scenario where the winner equivalences with this lead group algorithms can be discarded.

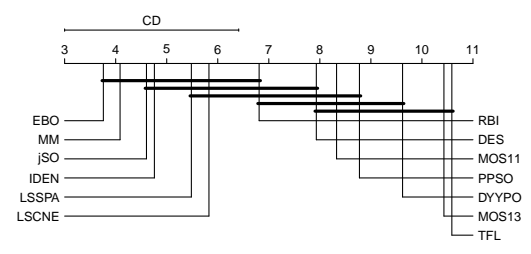

(a) CD plot Dim10

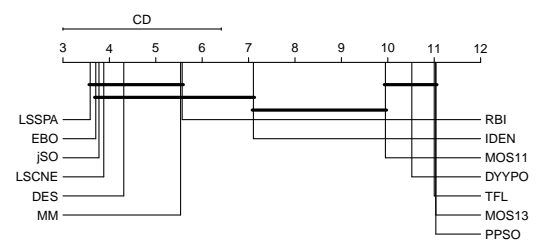

(c) CD plot Dim50

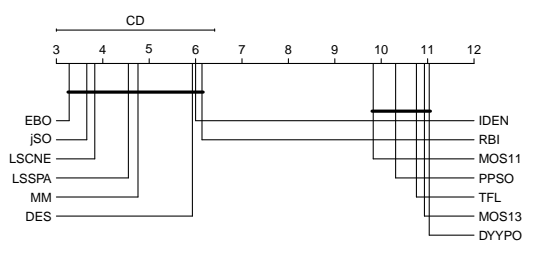

(b) CD plot Dim30

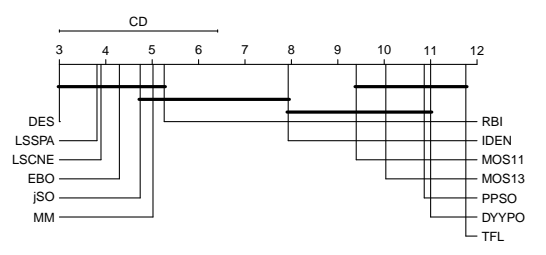

(d) CD plot Dim100

Figure 6: Plots of Critical Differences

In the Bayesian paradigm, after having rejected the equivalence of all the mean ranks of the algorithms with the Friedman tests, we repeatedly perform the Bayesian Signed-Rank for every pair of algorithms in each dimension scenario. The results are summarised in Figures 7-10. Especially in lower dimensions, there is less certainty than there was in the non-parametric analysis concerning the dominance of an algorithm over the other in each comparison, although 
from the Bayesian perspective we can state where there is a tie between a pair of algorithms and the direction of the difference, while the equivalence with NHST cannot be assured. The tiles for each column and row represent the comparison between the two algorithms. The colour depends on the result of the comparison, indicating if the greater probability belongs to the region of an algorithm or the rope. The probability of this hypothesis is written in the tile as well as represented in the opacity of the colour, to highlight the greater probabilities.

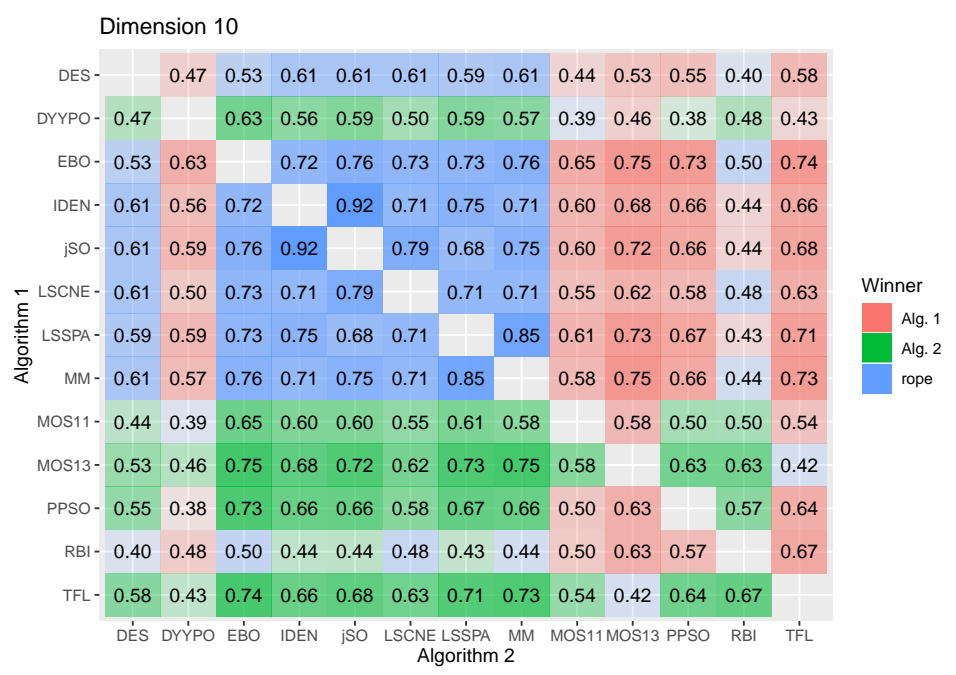

Figure 7: Bayesian Signed-Rank Dim10

As the error obtained in the competition increases, more comparisons are marked as significant and less ties between algorithms are detected. In Figure 7 we see how in the comparison of the first classified algorithms the most probable situation is a tie. This group starts to win with a greater probability in the 30 Dimension scenario (Figure 8), while the ties persist within the lead group. 


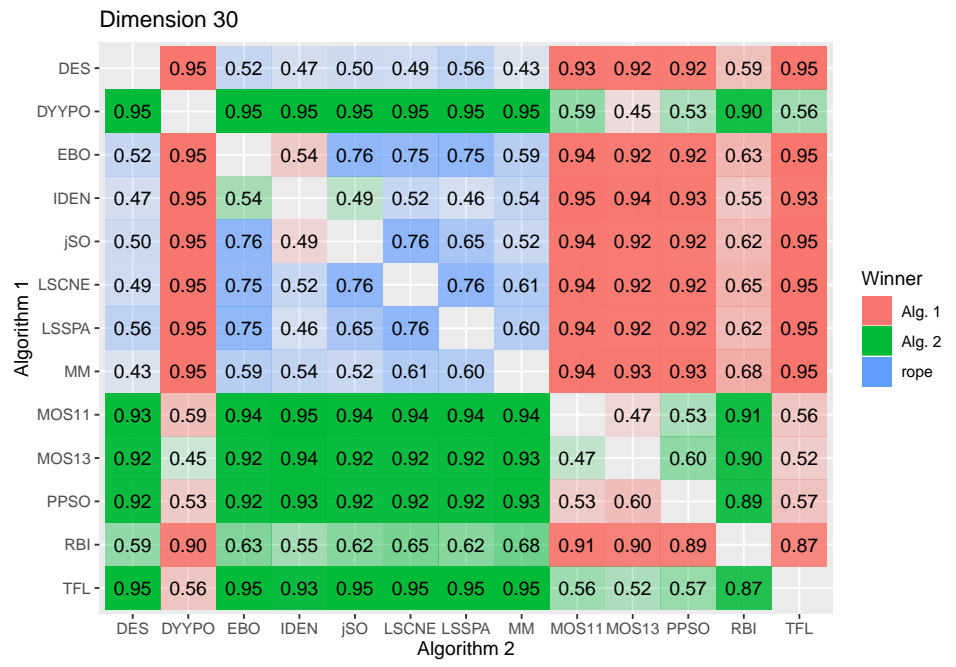

Figure 8: Bayesian Signed-Rank Dim30

The results of the 50 dimension scenario, shown in Figure 9, coincide with the conclusions obtained in the non-parametric analysis. In this scenario the lead group is reduced to EBO, jSO, LSCNE and DES, and the probabilities of the ties are lower than in previous scenarios. Finally in 100 dimension scenario, DES wins in the comparisons with all the remaining algorithms, with probabilities between 0.59 in the comparison versus LSCNE and 0.97 versus PPSO.

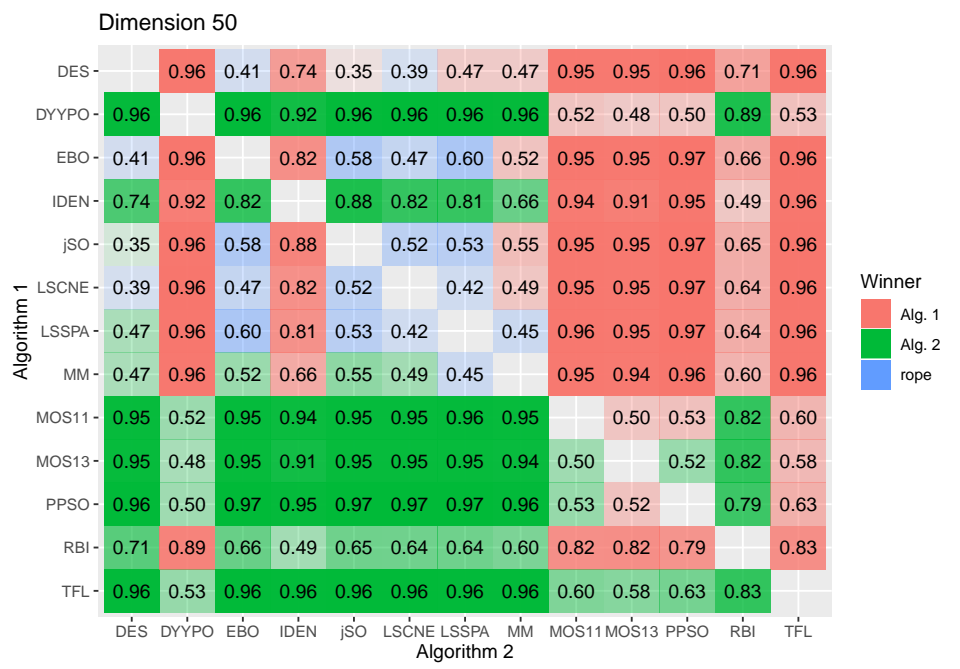

Figure 9: Bayesian Signed-Rank Dim50 


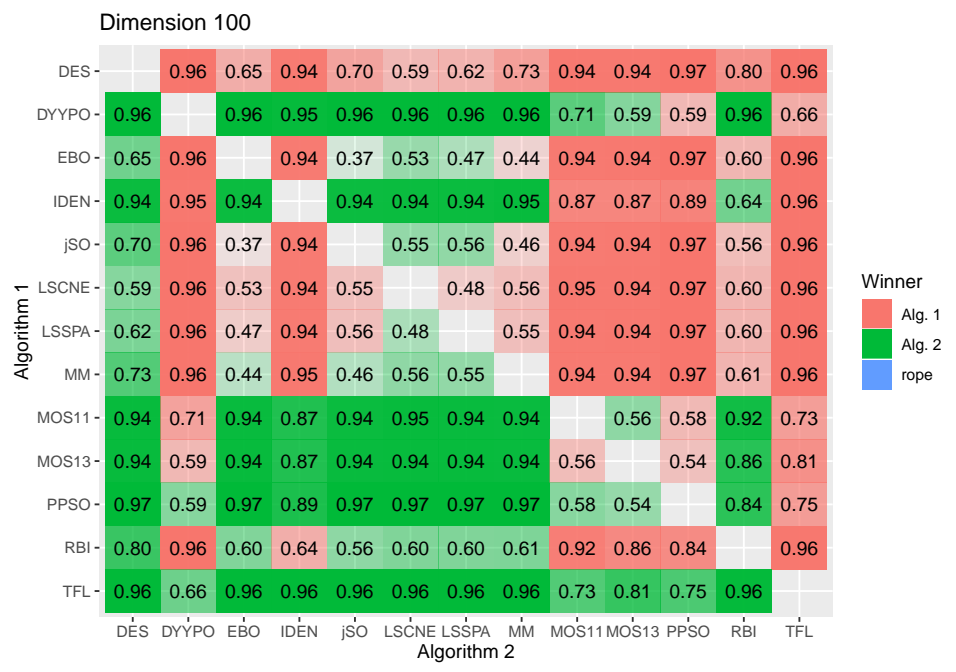

Figure 10: Bayesian Signed-Rank Dim100

\section{Discussion and Lessons Learnt}

In this section we include some considerations about the use of the tests described in previous sections and other proposed tests.

Criticisms on the $p$-value The criticisms made regarding the $p$-value and NHST are not limited to the Evolutionary Optimisation field or even Computer Science 92], but occur more frequently in other research fields, like psychology [93] or neuroscience 94].

A recent Nature paper 95] warns about the common mistakes in the interpretation about the meaning of the $p$-value, especially the statements about the alleged "no difference" between the groups when the null hypothesis is not rejected. This is one of the most direct and powerful arguments for the promotion of the use of the Bayesian tests, as the posterior distribution reflects the behaviour of the parameter of interest, and it is more difficult to conclude that the algorithms' performance is the same if it is not reflected in the plots of the distribution. The other main practical criticism is related to the researcher's intention and the effect size, as an elevated number of samples could derive in the rejection of the null hypothesis even with a tiny effect size. The Bayesian approach is not affected in the same way, as increasing the number of samples should be reflected in a posterior distribution closer to the underlying one.

Another controversial aspect of the NHST is their performance in a dichotomous way in order to determine the result of the experiment. However, these criticisms are not restricted to the frequentist paradigm and also affects the Bayesian perspective. Similar opinions appeared in ASA's statement [96], which represents a major setback to the use of NHST. They do recognise that to obtain 
reliable and repeatable results, diverse summaries of the data must be offered and the underlying process must be clearly understood and none of them can be substituted with a single index. This association now makes some proposals and gives some suggestions to avoid these pitfalls [97, like not asserting "statistically significant", accepting uncertainty or being more open to sharing the data and procedures.

Criticisms on the Bayesian paradigm Bayesian paradigm has a clear drawback of more complex tests and results, which causes this kind of test to be used less in experimentation. Moreover, some criticisms made regarding NHST and the decisions that depend on the researcher's intention, like the $\alpha$ value or the number of samples, remain in the Bayesian paradigm.

These decisions are the family and parameters from the prior distribution. The Bayesian tests described in this paper also contain the rope parameter for the equivalence of two algorithms, whose bounds are set to 0.01 in the original proposal for a classification scenario. Opting to adjust the results using Bayesian inference, selecting the prior distribution and making statements using this adjustment would lead to a model that could have a low representation of the data. Then a special consideration should be made in order to select a representative prior, with less information concerning the parameter of interest.

Frequentist and Bayesian Tests Relation Although there is a clear difference between the frequentist and the Bayesian paradigms, we could still hope to see a relationship between the results of both kinds of tests [98. In this line of argument, Couso et al. [99] built a frequentist test that simulates the behavior of the IDP test presented in subsection 5.2 for when the underlying distribution satisfies some properties. This study shows that in some circumstances the imprecise scenario using a set of prior distributions suggested from the Bayesian perspective is reflected in the distribution of $p$-values in the frequentist version.

Therefore, there is not a single way of obtaining conclusions, and they are not exempted from possible misuses or misunderstandings. The proliferation of the statistical tests and their application in erroneous circumstances can lead to spurious conclusions. Thus, the use of different tests can help to put the results in context. These considerations are independent of the guidelines for the experimentation in the field of evolutionary optimisation. The algorithms involved in the comparison should represent the state-of-the-art and the used benchmark should be relevant in the field of study.

\section{Conclusions}

This work contains an exhaustive set of statistical procedures and examples of their use in the comparison of results from experimental studies of optimisation algorithms, specifically in the scenario of a competition. In this paper we have described a broad set of methods, from basic non-parametric tests such 
as the Binomial Sign test to recently proposed Bayesian techniques such as Imprecise Dirichlet Process, with tools that can help researchers to complete their experimental study.

In this paper, we undertake an extensive statistical analysis of the results of the CEC '17 Special Session and Competition on Single-Objective Real Parameter Optimisation. The statistical analysis of the competition results provides different results according to the test used in different circumstances. Then, we have used this case of study to make some recommendations about which test is the most appropriate to each situation and how to proceed with the information that we get from them. Although the used paradigm depends on the intention of the researchers, they should consider using parametric tests only if the normality and homoscedasticity prerequisites are fulfilled. We have described some tests for the comparison of two algorithms, and the procedure when more algorithms are involved in the comparison, with specific tests for this purpose and some post-hoc procedures that indicate where the differences are.

Finally, we encourage the joint use of non-parametric and Bayesian tests in order to obtain a complete perspective of the comparison of the algorithms' results. While non-parametric tests can provide significant results when there is a difference between the compared algorithms, in some circumstances these tests do not provide any valuable information and Bayesian tests can help to elucidate the real difference between them. Furthermore, the rNPBST package and the shinytests application implement both perspectives and the procedures described in this tutorial.

\section{Acknowledgements}

This work is supported by the Spanish Ministry of Economy, Industry and Competitiveness under the Spanish National Research Project TIN2017-89517P. J. Carrasco was supported by the Spanish Ministry of Science under the FPU Programme 998758-2016 and by a scholarship of initiation to research granted by the University of Granada

\section{References}

[1] M. Hellwig, H.-G. Beyer, Benchmarking evolutionary algorithms for single objective real-valued constrained optimization - A critical review, Swarm and Evolutionary Computation 44 (2019) 927-944. doi:10.1016/ j.swevo.2018.10.002.

[2] J. Demšar, On the appropriateness of statistical tests in machine learning, in: Workshop on Evaluation Methods for Machine Learning in Conjunction with ICML, 2008, p. 65.

[3] D. J. Sheskin, Handbook of Parametric and Nonparametric Statistical Procedures, crc Press, 2003. 
[4] S. García, D. Molina, M. Lozano, F. Herrera, A study on the use of non-parametric tests for analyzing the evolutionary algorithms' behaviour: A case study on the CEC'2005 Special Session on Real Parameter Optimization, J. Heuristics 15 (6) (2009) 617-644. doi:10.1007/ s10732-008-9080-4.

[5] J. Derrac, S. García, S. Hui, P. N. Suganthan, F. Herrera, Analyzing convergence performance of evolutionary algorithms: A statistical approach, Information Sciences 289 (2014) 41-58.

[6] D. Berrar, Confidence curves: An alternative to null hypothesis significance testing for the comparison of classifiers, Machine Learning 106 (6) (2017) 911-949.

[7] A. Gelman, J. B. Carlin, H. S. Stern, D. B. Rubin, Bayesian Data Analysis, Second Edition (Chapman \& Hall/CRC Texts in Statistical Science), 2nd Edition, Chapman and Hall/CRC, 2003, published: Hardcover.

[8] A. Benavoli, G. Corani, J. Demšar, M. Zaffalon, Time for a Change: A Tutorial for Comparing Multiple Classifiers Through Bayesian Analysis, Journal of Machine Learning Research 18 (77) (2017) 1-36.

[9] N. Japkowicz, M. Shah (Eds.), Evaluating Learning Algorithms: A Classification Perspective, Cambridge University Press, 2011.

[10] P. Odile, Statistical Tests of Nonparametric Hypotheses: Asymptotic Theory, World Scientific, 2013.

[11] J. D. Gibbons, S. Chakraborti, Nonparametric Statistical Inference, CRC Press, 2010.

[12] M. J. Campbell, M. J. Gardner, Statistics in Medicine: Calculating confidence intervals for some non-parametric analyses, British medical journal (Clinical research ed.) 296 (6634) (1988) 1454.

[13] N. Awad, M. Ali, J. Liang, B. Qu, P. Suganthan, Problem Definitions and Evaluation Criteria for the CEC 2017 Special Session and Competition on Single Objective Real - Parameter Numerical Optimization, Tech. rep., NTU, Singapore (2016).

[14] J. Carrasco, S. García, M. del Mar Rueda, F. Herrera, rNPBST: An R Package Covering Non-parametric and Bayesian Statistical Tests, in: F. J. Martínez de Pisón, R. Urraca, H. Quintián, E. Corchado (Eds.), Hybrid Artificial Intelligent Systems: 12th International Conference, HAIS 2017, La Rioja, Spain, June 21-23, 2017, Proceedings, Springer International Publishing, Cham, 2017, pp. 281-292. doi:10.1007/978-3-319-59650-1_ 24.

[15] J. Demšar, Statistical comparisons of classifiers over multiple data sets, Journal of Machine learning research 7 (Jan) (2006) 1-30. 
[16] S. W. Looney, A statistical technique for comparing the accuracies of several classifiers, Pattern Recognition Letters 8 (1) (1988) 5-9. doi:10.1016/ 0167-8655(88) 90016-5.

[17] T. G. Dietterich, Approximate statistical tests for comparing supervised classification learning algorithms, Neural computation 10 (7) (1998) 18951923.

[18] E. Alpaydin, Combined 5 x 2 cv F Test for Comparing Supervised Classification Learning Algorithms, Neural Computation 11 (8) (1999) 1885-1892. doi:10.1162/089976699300016007.

[19] P. Castillo-Valdivieso, J. Merelo, A. Prieto, I. Rojas, G. Romero, Statistical analysis of the parameters of a neuro-genetic algorithm, IEEE Transactions on Neural Networks 13 (6) (2002) 1374-1394. doi:10.1109/tnn.2002. 804281.

[20] J. Pizarro, E. Guerrero, P. L. Galindo, Multiple comparison procedures applied to model selection, Neurocomputing 48 (1) (2002) 155-173.

[21] C. Nadeau, Y. Bengio, Inference for the generalization error, Machine Learning 52 (3) (2003) 239-281.

[22] D. Chen, X. Cheng, Statistical Comparisons of Multiple Classifiers, in: Proceedings of the International Conference on Machine Learning; Models, Technologies and Applications. MLMTA'03, June 23 - 26, 2003, Las Vegas, Nevada, USA, 2003, pp. 97-101.

[23] A. Czarn, C. MacNish, K. Vijayan, B. Turlach, R. Gupta, Statistical exploratory analysis of genetic algorithms, IEEE Transactions on Evolutionary Computation 8 (4) (2004) 405-421.

[24] C. S. Moskowitz, M. S. Pepe, Comparing the predictive values of diagnostic tests: Sample size and analysis for paired study designs, Clinical Trials 3 (3) (2006) 272-279.

[25] O. T. Yildiz, E. Alpaydin, Ordering and finding the best of K gt; 2 supervised learning algorithms, IEEE Transactions on Pattern Analysis and Machine Intelligence 28 (3) (2006) 392-402. doi:10.1109/TPAMI .2006.61.

[26] M. D. Smucker, J. Allan, B. Carterette, A comparison of statistical significance tests for information retrieval evaluation, in: Proceedings of the Sixteenth ACM Conference on Conference on Information and Knowledge Management, ACM, 2007, pp. 623-632.

[27] S. Garcia, F. Herrera, An Extension on"Statistical Comparisons of Classifiers over Multiple Data Sets"for all Pairwise Comparisons, Journal of Machine Learning Research 9 (Dec) (2008) 2677-2694. 
[28] O. Aslan, O. T. Yıldız, E. Alpaydın, Statistical comparison of classifiers using area under the roc curve (2009).

[29] S. García, A. Fernández, J. Luengo, F. Herrera, A study of statistical techniques and performance measures for genetics-based machine learning: Accuracy and interpretability, Soft Computing 13 (10) (2009) 959.

[30] J. Luengo, S. García, F. Herrera, A study on the use of statistical tests for experimentation with neural networks: Analysis of parametric test conditions and non-parametric tests, Expert Systems with Applications 36 (4) (2009) 7798-7808.

[31] S. García, A. Fernández, J. Luengo, F. Herrera, Advanced nonparametric tests for multiple comparisons in the design of experiments in computational intelligence and data mining: Experimental analysis of power, Inf. Sci. 180 (10) (2010) 2044-2064. doi:10.1016/j.ins.2009.12.010.

[32] P. H. Westfall, J. F. Troendle, G. Pennello, Multiple McNemar Tests, Biometrics 66 (4) (2010) 1185-1191. doi:10.1111/j.1541-0420.2010. 01408.x.

[33] J. Rodriguez, A. Perez, J. Lozano, Sensitivity Analysis of k-Fold Cross Validation in Prediction Error Estimation, IEEE Transactions on Pattern Analysis and Machine Intelligence 32 (3) (2010) 569-575. doi:10.1109/ TPAMI.2009.187.

[34] M. Ojala, G. C. Garriga, Permutation tests for studying classifier performance, Journal of Machine Learning Research 11 (Jun) (2010) 1833-1863.

[35] E. G. Carrano, E. F. Wanner, R. H. C. Takahashi, A Multicriteria Statistical Based Comparison Methodology for Evaluating Evolutionary Algorithms, IEEE Transactions on Evolutionary Computation 15 (6) (2011) 848-870. doi:10.1109/TEVC.2010.2069567.

[36] J. Derrac, S. García, D. Molina, F. Herrera, A practical tutorial on the use of nonparametric statistical tests as a methodology for comparing evolutionary and swarm intelligence algorithms, Swarm and Evolutionary Computation 1 (1) (2011) 3 - 18.

[37] B. Trawiński, M. Smętek, Z. Telec, T. Lasota, Nonparametric statistical analysis for multiple comparison of machine learning regression algorithms, International Journal of Applied Mathematics and Computer Science 22 (4) (Jan. 2012). doi:10.2478/v10006-012-0064-z.

[38] A. Ulaş, O. T. Yıldız, E. Alpaydın, Cost-conscious comparison of supervised learning algorithms over multiple data sets, Pattern Recognition 45 (4) (2012) 1772-1781. doi:10.1016/j.patcog.2011.10.005 
[39] O. Irsoy, O. T. Yildiz, E. Alpaydin, Design and Analysis of Classifier Learning Experiments in Bioinformatics: Survey and Case Studies, IEEE/ACM Transactions on Computational Biology and Bioinformatics 9 (6) (2012) 1663-1675. doi:10.1109/TCBB.2012.117.

[40] A. Lacoste, F. Laviolette, M. Marchand, Bayesian comparison of machine learning algorithms on single and multiple datasets, in: Artificial Intelligence and Statistics, 2012, pp. 665-675.

[41] K. H. Brodersen, C. Mathys, J. R. Chumbley, J. Daunizeau, C. S. Ong, J. M. Buhmann, K. E. Stephan, Bayesian mixed-effects inference on classification performance in hierarchical data sets, Journal of Machine Learning Research 13 (Nov) (2012) 3133-3176.

[42] O. T. Ylldı, E. Alpaydın, Statistical Tests Using Hinge/ $\epsilon$-Sensitive Loss, in: E. Gelenbe, R. Lent (Eds.), Computer and Information Sciences III, Springer London, London, 2013, pp. 153-160. doi:10.1007/ 978-1-4471-4594-3_16.

[43] B. Bostanci, E. Bostanci, An Evaluation of Classification Algorithms Using Mc Nemar's Test, in: J. C. Bansal, P. K. Singh, K. Deep, M. Pant, A. K. Nagar (Eds.), Proceedings of Seventh International Conference on Bio-Inspired Computing: Theories and Applications (BIC-TA 2012), Vol. 201, Springer India, India, 2013, pp. 15-26. doi:10.1007/ 978-81-322-1038-2_2.

[44] J. Otero, L. Sánchez, I. Couso, A. Palacios, Bootstrap analysis of multiple repetitions of experiments using an interval-valued multiple comparison procedure, Journal of Computer and System Sciences 80 (1) (2014) 88 100. doi:http://dx.doi.org/10.1016/j.jcss.2013.03.009.

[45] W. Yu, W. Ruibo, J. Huichen, L. Jihong, Blocked 3x2 Cross-Validated $t$ -Test for Comparing Supervised Classification Learning Algorithms, Neural Computation 26 (1) (2014) 208-235. doi:10.1162/NECO_a_00532.

[46] S. García, J. Derrac, S. Ramírez-Gallego, F. Herrera, On the statistical analysis of the parameters' trend in a machine learning algorithm, Progress in Artificial Intelligence 3 (1) (2014) 51-53. doi:10.1007/ s13748-014-0043-8.

[47] A. Benavoli, G. Corani, F. Mangili, M. Zaffalon, F. Ruggeri, A Bayesian Wilcoxon signed-rank test based on the Dirichlet process, in: Proceedings of the 31th International Conference on Machine Learning, ICML 2014, Beijing, China, 21-26 June 2014, 2014, pp. 1026-1034.

[48] A. Benavoli, F. Mangili, F. Ruggeri, M. Zaffalon, Imprecise Dirichlet Process With Application to the Hypothesis Test on the Probability That $X$ $\leq Y$, Journal of Statistical Theory and Practice 9 (3) (2015) 658-684. doi:10.1080/15598608.2014.985997. 
[49] G. Corani, A. Benavoli, A Bayesian approach for comparing cross-validated algorithms on multiple data sets, Machine Learning 100 (2-3) (2015) 285304. doi:10.1007/s10994-015-5486-z.

[50] A. Benavoli, C. P. de Campos, Statistical Tests for Joint Analysis of Performance Measures, in: Advanced Methodologies for Bayesian Networks - Second International Workshop, AMBN 2015, Yokohama, Japan, November 16-18, 2015. Proceedings, 2015, pp. 76-92. doi:10.1007/ 978-3-319-28379-1_6

[51] A. Benavoli, G. Corani, F. Mangili, M. Zaffalon, A Bayesian nonparametric procedure for comparing algorithms, in: Proceedings of the 32nd International Conference on Machine Learning, ICML 2015, Lille, France, 6-11 July 2015, 2015, pp. 1264-1272.

[52] Y. Wang, J. Li, Y. Li, R. Wang, X. Yang, Confidence Interval for F_1 Measure of Algorithm Performance Based on Blocked 3x2 Cross-Validation, IEEE Transactions on Knowledge and Data Engineering 27 (3) (2015) 651659. doi:10.1109/TKDE.2014.2359667.

[53] J. Perolat, I. Couso, K. Loquin, O. Strauss, Generalizing the Wilcoxon rank-sum test for interval data, International Journal of Approximate Reasoning 56 (2015) 108-121. doi:10.1016/j.ijar.2014.08.001.

[54] P. K. Singh, R. Sarkar, M. Nasipuri, Statistical validation of multiple classifiers over multiple datasets in the field of pattern recognition, International Journal of Applied Pattern Recognition 2 (1) (2015) 1-23.

[55] L. Gondara, Classifier comparison using precision, arXiv preprint arXiv:1609.09471 (2016).

[56] G. Corani, A. Benavoli, J. Demšar, F. Mangili, M. Zaffalon, Statistical comparison of classifiers through Bayesian hierarchical modelling, Machine Learning (2016) 1-21.

[57] R. Eisinga, T. Heskes, B. Pelzer, M. Te Grotenhuis, Exact p-values for pairwise comparison of Friedman rank sums, with application to comparing classifiers, BMC Bioinformatics 18 (1) (Dec. 2017). doi:10.1186/ s12859-017-1486-2.

[58] Z. Yu, Z. Wang, J. You, J. Zhang, J. Liu, H. Wong, G. Han, A New Kind of Nonparametric Test for Statistical Comparison of Multiple Classifiers Over Multiple Datasets, IEEE Transactions on Cybernetics 47 (12) (2017) 4418-4431. doi:10.1109/TCYB.2016.2611020.

[59] T. Eftimov, P. Korošec, B. K. Seljak, Comparing multi-objective optimization algorithms using an ensemble of quality indicators with deep statistical comparison approach, in: 2017 IEEE Symposium Series on Computational Intelligence (SSCI), 2017, pp. 1-8. doi:10.1109/SSCI.2017.8280910. 
[60] B. Calvo, J. Ceberio, J. A. Lozano, Bayesian Inference for Algorithm Ranking Analysis, in: Proceedings of the Genetic and Evolutionary Computation Conference Companion, GECCO '18, ACM, New York, NY, USA, 2018, pp. 324-325. doi:10.1145/3205651.3205658.

[61] F. Campelo, F. Takahashi, Sample size estimation for power and accuracy in the experimental comparison of algorithms, Journal of Heuristics 25 (2) (2019) 305-338, 00002. doi:10.1007/s10732-018-9396-7

[62] T. Eftimov, P. Korošec, A novel statistical approach for comparing metaheuristic stochastic optimization algorithms according to the distribution of solutions in the search space, Information Sciences 489 (2019) 255-273, 00000. doi:10.1016/j.ins.2019.03.049.

[63] F. Pesarin, L. Salmaso, Permutation Tests for Complex Data: Theory, Applications and Software, John Wiley \& Sons, 2010.

[64] D. W. Nordstokke, B. D. Zumbo, A new nonparametric Levene test for equal variances, Psicológica 31 (2) (2010).

[65] E. Kasuya, Wilcoxon signed-ranks test: Symmetry should be confirmed before the test, Animal Behaviour 79 (3) (2010) 765-767. doi:10.1016/ j. anbehav.2009.11.019.

[66] W. J. Dixon, A. M. Mood, The statistical sign test, Journal of the American Statistical Association 41 (236) (1946) 557-566.

[67] F. Wilcoxon, Individual Comparisons by Ranking Methods, Biometrics Bulletin 1 (6) (1945) 80-83. doi:10.2307/3001968.

[68] W. J. Conover, R. L. Iman, Rank transformations as a bridge between parametric and nonparametric statistics, The American Statistician 35 (3) (1981) 124-129.

[69] A. L. Rhyne, R. G. D. Steel, Tables for a Treatments Versus Control Multiple Comparisons Sign Test, Technometrics 7 (3) (1965) 293-306.

[70] R. L. Iman, J. M. Davenport, Approximations of the critical region of the fbietkan statistic, Communications in Statistics - Theory and Methods 9 (6) (1980) 571-595, 00709. doi:10.1080/03610928008827904.

[71] J. L. Hodges, E. L. Lehmann, Rank Methods for Combination of Independent Experiments in Analysis of Variance, The Annals of Mathematical Statistics 33 (2) (1962) 482-497. doi:10.1214/aoms/1177704575

[72] D. Quade, Rank analysis of covariance, Journal of the American Statistical Association 62 (320) (1967) 1187-1200. 
[73] D. Berrar, J. A. Lozano, Significance tests or confidence intervals: Which are preferable for the comparison of classifiers?, Journal of Experimental \& Theoretical Artificial Intelligence 25 (2) (2013) 189-206. doi:10.1080/ $0952813 X .2012 .680252$.

[74] J. Seldrup, C. Lentner, K. Diem, Geigy Scientific Tables: Introduction to Statistics, Statistical Tables, Mathematical Formulae, Ciba-Geigy, 1982.

[75] P. I. Good, J. W. Hardin, Common Errors in Statistics (and How to Avoid Them), John Wiley \& Sons, 2012.

[76] J. K. Kruschke, Bayesian data analysis, Wiley Interdisciplinary Reviews: Cognitive Science 1 (5) (2010) 658-676.

[77] G. Willems, G. Pison, P. J. Rousseeuw, S. Van Aelst, A robust Hotelling test, Metrika 55 (1) (2002) 125-138, 00058. doi:10.1007/s001840200192

[78] J. A. Villasenor Alva, E. G. Estrada, A generalization of Shapiro-Wilk's test for multivariate normality, Communications in Statistics-Theory and Methods 38 (11) (2009) 1870-1883, 00126.

[79] C. P. de Campos, A. Benavoli, Joint Analysis of Multiple Algorithms and Performance Measures, New Generation Computing 35 (1) (2016) 69-86. doi:10.1007/s00354-016-0005-8.

[80] A. Kumar, R. K. Misra, D. Singh, Improving the local search capability of Effective Butterfly Optimizer using Covariance Matrix Adapted Retreat Phase, in: 2017 IEEE Congress on Evolutionary Computation (CEC), 2017, pp. 1835-1842. doi:10.1109/CEC.2017.7969524.

[81] J. Brest, M. S. Maučec, B. Bošković, Single objective real-parameter optimization: Algorithm jSO, in: Evolutionary Computation (CEC), 2017 IEEE Congress On, IEEE, 2017, pp. 1311-1318.

[82] N. H. Awad, M. Z. Ali, P. N. Suganthan, Ensemble sinusoidal differential covariance matrix adaptation with Euclidean neighborhood for solving CEC2017 benchmark problems, in: Evolutionary Computation (CEC), 2017 IEEE Congress On, IEEE, 2017, pp. 372-379.

[83] A. W. Mohamed, A. A. Hadi, A. M. Fattouh, K. M. Jambi, LSHADE with semi-parameter adaptation hybrid with CMA-ES for solving CEC 2017 benchmark problems, in: Evolutionary Computation (CEC), 2017 IEEE Congress On, IEEE, 2017, pp. 145-152.

[84] D. Jagodziński, J. Arabas, A differential evolution strategy, in: Evolutionary Computation (CEC), 2017 IEEE Congress On, IEEE, 2017, pp. $1872-1876$. 
[85] K. M. Sallam, S. M. Elsayed, R. A. Sarker, D. L. Essam, Multi-method based orthogonal experimental design algorithm for solving CEC2017 competition problems, in: Evolutionary Computation (CEC), 2017 IEEE Congress On, IEEE, 2017, pp. 1350-1357.

[86] P. Bujok, J. Tvrdík, Enhanced individual-dependent differential evolution with population size adaptation, in: Evolutionary Computation (CEC), 2017 IEEE Congress On, IEEE, 2017, pp. 1358-1365.

[87] R. Biedrzycki, A version of IPOP-CMA-ES algorithm with midpoint for CEC 2017 single objective bound constrained problems, in: Evolutionary Computation (CEC), 2017 IEEE Congress On, IEEE, 2017, pp. 1489-1494.

[88] A. LaTorre, J.-M. Peña, A comparison of three large-scale global optimizers on the CEC 2017 single objective real parameter numerical optimization benchmark, in: Evolutionary Computation (CEC), 2017 IEEE Congress On, IEEE, 2017, pp. 1063-1070.

[89] A. Tangherloni, L. Rundo, M. S. Nobile, Proactive Particles in Swarm Optimization: A settings-free algorithm for real-parameter single objective optimization problems, in: Evolutionary Computation (CEC), 2017 IEEE Congress On, IEEE, 2017, pp. 1940-1947.

[90] D. Maharana, R. Kommadath, P. Kotecha, Dynamic Yin-Yang Pair Optimization and its performance on single objective real parameter problems of CEC 2017, in: Evolutionary Computation (CEC), 2017 IEEE Congress On, IEEE, 2017, pp. 2390-2396.

[91] R. Kommadath, P. Kotecha, Teaching Learning Based Optimization with focused learning and its performance on CEC2017 functions, in: Evolutionary Computation (CEC), 2017 IEEE Congress On, IEEE, 2017, pp. 2397-2403.

[92] D. Berrar, W. Dubitzky, On the Jeffreys-Lindley Paradox and the Looming Reproducibility Crisis in Machine Learning, in: Data Science and Advanced Analytics (DSAA), 2017 IEEE International Conference On, IEEE, 2017, pp. $334-340$.

[93] S. L. Chow, Précis of statistical significance: Rationale, validity, and utility, Behavioral and brain sciences 21 (2) (1998) 169-194.

[94] F. Melinscak, L. Montesano, Beyond p-values in the evaluation of brain-computer interfaces: A Bayesian estimation approach, Journal of neuroscience methods 270 (2016) 30-45.

[95] V. Amrhein, S. Greenland, B. McShane, Scientists rise up against statistical significance, Nature 567 (7748) (2019) 305, 00002. doi:10.1038/ d41586-019-00857-9. 
[96] R. L. Wasserstein, N. A. Lazar, The ASA's Statement on p-Values: Context, Process, and Purpose, The American Statistician 70 (2) (2016) 129 133. doi:10.1080/00031305.2016.1154108.

[97] R. L. Wasserstein, A. L. Schirm, N. A. Lazar, Moving to a World Beyond "p < 0.05", The American Statistician 73 (sup1) (2019) 1-19, 00003. doi: 10.1080/00031305.2019.1583913.

[98] I. R. Silva, On the correspondence between frequentist and Bayesian tests, Communications in Statistics - Theory and Methods 47 (14) (2018) 34773487. doi:10.1080/03610926.2017.1359296.

[99] I. Couso, A. Álvarez-Caballero, L. Sánchez, Reconciling Bayesian and Frequentist Tests: The Imprecise Counterpart, in: A. Antonucci, G. Corani, I. Couso, S. Destercke (Eds.), Proceedings of the Tenth International Symposium on Imprecise Probability: Theories and Applications, Vol. 62 of Proceedings of Machine Learning Research, PMLR, 2017, pp. 97-108. 